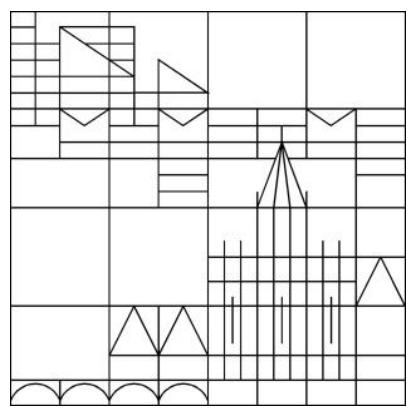

\title{
Hyperbolic compressible Navier-Stokes equations
}

\author{
Yuxi $\mathrm{Hu}$ \\ Reinhard Racke
}

Konstanzer Schriften in Mathematik

Nr. 384, Oktober 2019

ISSN 1430-3558 



\title{
HYPERBOLIC COMPRESSIBLE NAVIER-STOKES EQUATIONS
}

\author{
YUXI HU AND REINHARD RACKE
}

\begin{abstract}
We consider the non-isentropic compressible Navier-Stokes equations with hyperbolic heat conduction and a law for the stress tensor which is modified correspondingly by Maxwell's law. These two relaxations, turning the whole system into a hyperbolic one, are not only treated simultaneously, but are also considered in a version having Galilean invariance. For this more complicated relaxed system, the global well-posedness is proved for small data. Moreover, for vanishing relaxation parameters the solutions are shown to converge to solutions of the classical system.
\end{abstract}

Keywords:

AMS classification code: $35 \mathrm{~B} 25,76 \mathrm{~N} 10$

\section{INTRODUCTION}

We consider the system of non-isentropic compressible Navier-Stokes equations in $\mathbb{R} \times[0, \infty)$ in the following hyperbolic form:

$$
\left\{\begin{array}{l}
\rho_{t}+(\rho u)_{x}=0 \\
\rho u_{t}+\rho u u_{x}+p_{x}=S_{x} \\
\rho e_{t}+\rho u e_{x}+p u_{x}+q_{x}=S u_{x}
\end{array}\right.
$$

with

$$
\tau_{1}\left(q_{t}+u q_{x}\right)+q+\kappa \theta_{x}=0
$$

and

$$
\tau_{2}\left(S_{t}+u S_{x}\right)+S=\mu u_{x}
$$

Here, $\rho, u, p, S, e, \theta$ and $q$ represent fluid density, velocity, pressure, stress tensor, specific internal energy per unit mass, temperature and heat flux, respectively. The equations (1.1) are the consequence of conservation of mass, momentum and energy, respectively. $\kappa, \mu, \lambda$ are positive constants as well as the relaxation parameters $\tau_{1}$ and $\tau_{2}$.

We investigate the Cauchy problem for the functions

$$
(\rho, u, \theta, q, S): \mathbb{R} \times[0,+\infty) \rightarrow \mathbb{R}_{+} \times \mathbb{R} \times \mathbb{R}_{+} \times \mathbb{R} \times \mathbb{R}
$$

with initial condition

$$
(\rho(x, 0), u(x, 0), \theta(x, 0), q(x, 0), S(x, 0))=\left(\rho_{0}, u_{0}, \theta_{0}, q_{0}, S_{0}\right) .
$$

For $\tau_{1}=\tau_{2}=0$ we recover the classical non-isentropic compressible Navier-Stokes equations.

Neglecting the quadratic nonlinear terms $u q_{x}$ and $u S_{x}$ in (1.2) resp. (1.3), the case $\tau_{2}=0$, $\tau_{1}>0$ (Cattaneo law) has been studied in $\mathbf{R}^{n}, n \geq 1$, in our paper [15]. The case $\tau_{1}=0$, $\tau_{2}>0$ (Maxwell's law) in $\mathbf{R}^{n}, n=2,3$, was treated in [16]. In the latter a splitted version

Yuxi Hu, Department of Mathematics, China University of Mining and Technology, Beijing, 100083, P.R. China, yxhu86@163.com

Reinhard Racke, Department of Mathematics and Statistics, University of Konstanz, 78457 Konstanz, Germany, reinhard.racke@uni-konstanz.de. 
of the relaxation law involving two relaxation for different parts of the stress tensor, which was first proposed and treated by Yong [37] for the isentropic case, was considered. A similar revised Maxwell model was considered by Chakraborty \& Sader [1] for a compressible viscoelastic fluid (isentropic case), where one relaxation counts for the shear relaxation time, and the other counts for the compressional relaxation time. The importance of this model for describing high frequency limits is underlined together with the presentation of numerical experiments. The authors conclude that it provides a general formalism with which to characterize the fluid-structure interaction of nanoscale mechanical devices vibrating in simple liquids.

Now, here, we have as main new contributions:

* Taking into account the two nonlinear terms in (1.2), (1.3). This is motivated from requiring Galilean invariance of the system as suggested by Christov \& Jordan [5].

* Discussion the two relaxations - for heat and stress - simultaneously.

* Deriving a global well-posedness result for small data as well as the rigorous limit as $\tau:=\tau_{1}=$ $\tau_{2} \rightarrow 0$, i.e. the convergence to the classical Navier-Stokes equations, giving convergence rates in terms of powers of the relaxation parameter $\tau$.

For $\tau=0$, the relaxed system (1.1)-(1.3) turns into the classical Newtonian compressible NavierStokes system. For the latter, because of its physical importance and mathematical challenges, the well-posedness has been widely studied, see $[3,4,10,11,12,17,18,19,21,22,23,24,25,31,35]$. In particular, the local existence and uniqueness of smooth solutions was established by Serrin [31] and Nash [25] for initial data far away from vacuum. Later, Matsumura and Nishida [23] got global smooth solutions for small initial data without vacuum. For large data, Xin [35], Cho and Jin [3] showed that smooth solutions must blow up in finite time if the initial data has a vacuum state.

One should note that it is not obvious that the results which hold for the classical systems also hold for the relaxed system. Indeed, and for example, Hu and Wang [14] showed that, for the one-dimensional isentropic compressible Navier-Stokes system, classical solutions exist globally for arbitrary large initial data, while solutions blow up in finite time for some large initial data for the corresponding relaxed system. A similar qualitative change was observed before for certain thermoelastic systems, where the non-relaxed system is exponentially stable, while the relaxed one is not, see Quintanilla and Racke resp. Fernández Sare and Muñoz Rivera [26, 8] for plates, and Fernández Sare and Racke [9] for Timoshenko beams.

For incompressible Navier-Stokes equations the relaxation, without nonlinearity in (1.3), has been discussed by Racke \& Saal [27, 28] and Schöwe [29, 30] proving global well-posedness for small data and rigorously investigating the singular limit as $\tau=\tau_{2} \rightarrow 0$.

We assume the internal energy $e$ and pressure $p$ have the following form:

$$
e=c_{v} \theta+\frac{\tau_{1}}{\kappa \theta \rho} q^{2}+\frac{\tau_{2}}{2 \mu \rho} S^{2}
$$

and

$$
p=R \rho \theta-\frac{\tau_{1}}{2 \kappa \theta} q^{2}-\frac{\tau_{2}}{2 \mu} S^{2}
$$

such that they satisfy the thermodynamic equation $\rho^{2} e_{\rho}=p-\theta p_{\theta}$.

The dependence on $q^{2}$ term of the internal energy is indicated in paper [6], where they rigorously prove that such constitutive equations are consistent with the second law of thermodynamics if and only if one use the relaxation equation (1.2), see also [2, 7, 33]. Since we also consider a relaxation for the stress tensor $S$, it is motivated, naturally, by [6] that the internal energy should also depend on $S$ in a quadratic form. Indeed, under the above constitutive laws, we have a dissipative entropy for our system (1.1)-(1.3), see Lemma 3.1, which implies the compatibility with the second law of thermodynamics.

The main results are the following. First, we have global existence for small data. 
Theorem 1.1. There exists $\varepsilon>0$ such that if

$$
\|\left(\rho_{0}-1, u_{0}, \theta_{0}-1, q_{0}, S_{0} \|_{H^{2}}^{2}<\varepsilon^{2},\right.
$$

there exists a globally defined solution $(\rho, u, \theta, q, S)(x, t) \in \mathcal{C}^{1}([0,+\infty) \times \mathbb{R})$ to the initial value problem (1.1)-(1.4) satisfying

$$
\frac{3}{4} \leq \sup _{x, t}(\rho(x, t), \theta(x, t)) \leq \frac{5}{4}
$$

and

$$
\sup _{t \in[0, \infty)}\|(\rho-1, u, \theta-1, q, S)\|_{H^{2}}^{2} \leq C \|\left(\rho_{0}-1, u_{0}, \theta_{0}-1, q_{0}, S_{0} \|_{H^{2}}^{2} \leq C \varepsilon^{2}\right.
$$

where $C$ is a constant which is independent of $\varepsilon$. Moreover, the solution converges, uniformly in $x \in \mathbb{R}$ to the constant state $(1,0,1,0,0)$ as $t \rightarrow \infty$. Namely,

$$
\|(\rho-1, u, \theta-1, q, S)\|_{L^{\infty}}+\left\|\left(\rho_{x}, u_{x}, \theta_{x}, q_{x}, S_{x}\right)\right\|_{L^{2}} \rightarrow 0 \quad \text { as } \quad t \rightarrow \infty .
$$

Second, we have a description of the singular limit $\tau \rightarrow 0$ where we assume for simplicity $\tau_{1}=\tau_{2}=: \tau$. We also assume the compatibility condition on the initial data,

$$
S_{0}=\mu\left(u_{0}\right)_{x}, q_{0}=-\kappa\left(\theta_{0}\right)_{x}
$$

Let $\left(\rho^{\tau}, u^{\tau}, \theta^{\tau}, q^{\tau}, S^{\tau}\right)$ be solutions given by Theorem 1.1. Define

$$
T_{\tau}=\sup \left\{T>0 ;\left(\rho^{\tau}-1, u^{\tau}, \theta^{\tau}-1, q^{\tau}, S^{\tau}\right) \in C\left([0, T], H^{2}\right), \rho^{\tau}>0, \theta^{\tau}>0, \forall(x, t) \in \mathbb{R}^{n} \times[0, T]\right\} .
$$

Then we have

Theorem 1.2. Let $(\rho, u, \theta)$ be smooth solution to the classical compressible Navier-Stokes equations with $(\rho(x, 0), u(x, 0), \theta(x, 0))=\left(\rho_{0}, u_{0}, \theta_{0}\right)$ satisfying $\inf _{(x, t) \in \mathbb{R} \times\left[0, T_{*}\right]}(\rho(x, t), \theta(x, t))>0$ and

$$
\begin{array}{r}
(\rho-1) \in C\left(\left[0, T_{*}\right], H^{5}\right) \cap C^{1}\left(\left[0, T_{*}\right], H^{4}\right), \\
(u, \theta-1) \in C\left(\left[0, T_{*}\right], H^{5}\right) \cap C^{1}\left(\left[0, T_{*}\right], H^{3}\right),
\end{array}
$$

with $T_{*}>0$ be finite. Then, there exist constants $\tau_{0}$ and $C$ such that for $\tau \leq \tau_{0}$,

$$
\left\|\left(\rho^{\tau}, u^{\tau}, \theta^{\tau}\right)(t, \cdot)-(\rho, u, \theta)(t, \cdot)\right\|_{H^{2}} \leq C \tau,
$$

and

$$
\left\|\left(q^{\tau}+\kappa \theta_{x}, S^{\tau}-\mu u_{x}\right)\right\|_{H^{2}} \leq C \tau^{\frac{1}{2}},
$$

for all $t \in\left(0, \min \left(T_{*}, T_{\tau}\right)\right)$, and the constant $C$ is independent of $\tau$.

The $H^{5}$-regularity is needed to estimate some terms in the proof of Theorem 1.2, e.g. the terms $F_{i}$ there, see below.

The paper is organized as follows. In Section 2 we prove the local well-posedness, the global existence result Theorem 1.1 is proved in Section 3. The singular limit as $\tau \rightarrow 0$ is subject of Section 4, where Theorem 1.2 is proved.

Finally, we introduce some notation. $W^{m, p}=W^{m, p}\left(\mathbb{R}^{n}\right), 0 \leq m \leq \infty, 1 \leq p \leq \infty$, denotes the usual Sobolev space with norm $\|\cdot\|_{W^{m, p}}, H^{m}$ and $L^{p}$ stand for $W^{m, 2}(\Omega)$ resp. $W^{0, p}(\Omega)$. 


\section{Local Existence Theorem}

In this part, we establish the local existence theorem for system (1.1)-(1.4). We rewrite the system (1.1) as follows:

$$
\left\{\begin{array}{l}
\rho_{t}+(\rho u)_{x}=0 \\
\rho u_{t}+\rho u u_{x}+p_{\rho} \rho_{x}+p_{\theta} \theta_{x}+p_{q} q_{x}+\left(p_{S}-1\right) S_{x}=0 \\
\rho e_{\theta} \theta_{t}+\left(\rho u e_{\theta}-\frac{2 q}{\theta}\right) \theta_{x}+\theta p_{\theta} u_{x}+q_{x}=\frac{2 q^{2}}{\kappa \theta}+\frac{S^{2}}{\mu} \\
\tau_{1}\left(q_{t}+u q_{x}\right)+q+\kappa \theta_{x}=0 \\
\tau_{2}\left(S_{t}+u S_{x}\right)+S=\mu u_{x} .
\end{array}\right.
$$

For the derivation of equation $(2.1)_{3}$, we calculate

$$
\begin{aligned}
& \rho e_{t}=\rho e_{\theta} \theta_{t}+\rho e_{\rho} \rho_{t}+\rho e_{q} q_{t}+\rho e_{S} S_{t} \\
& =\rho e_{\theta} \theta_{t}+\rho e_{\rho} \rho_{t}+\rho \frac{2 \tau_{1}}{\kappa \theta \rho} q q_{t}+\rho \frac{\tau_{2}}{\mu \rho} S S_{t} \\
& =\rho e_{\theta} \theta_{t}+\rho e_{\rho} \rho_{t}+\frac{2}{\kappa \theta} q\left(-\tau_{1} u q_{x}-q-\kappa \theta_{x}\right)+\frac{S}{\mu}\left(-\tau_{2} u S_{x}-S+\mu u_{x}\right) \\
& =\rho e_{\theta} \theta_{t}-\frac{2 q}{\theta} \theta_{x}+\rho e_{\rho} \rho_{t}-\frac{2 \tau_{1}}{\kappa \theta} u q q_{x}-\frac{2}{\kappa \theta} q^{2}-\frac{\tau_{2}}{\mu} u S S_{x}-\frac{S^{2}}{\mu}+S u_{x},
\end{aligned}
$$

while

$$
\rho u e_{x}=\rho u\left(e_{\theta} \theta_{x}+e_{\rho} \rho_{x}+e_{q} q_{x}+e_{S} S_{x}\right)=\rho u e_{\theta} \theta_{x}+\rho u e_{\rho} \rho_{x}+\rho u \frac{2 \tau_{1}}{\kappa \theta \rho} q q_{x}+\rho u \frac{\tau_{2} S}{\mu \rho} S_{x} .
$$

So, combining the above equalities, we derive

$$
\rho e_{t}+\rho u e_{x}=\rho e_{\theta} \theta_{t}+\left(\rho u e_{\theta}-\frac{2 q}{\theta}\right) \theta_{x}+\rho e_{\rho}\left(\rho_{t}+u \rho_{x}\right)-\frac{2}{\kappa \theta} q^{2}-\frac{S^{2}}{\mu}+S u_{x} .
$$

On the other hand, by the thermodynamic equation, we have $p u_{x}=\left(\theta p_{\theta}+\rho^{2} e_{\rho}\right) u_{x}=\theta p_{\theta} u_{x}+$ $\rho^{2} e_{\rho} u_{x}$. Combining these calculations and using the mass equation $(1.1)_{1}$, we derive equation $(2.1)_{3}$.

Note that the system (2.1) is non-symmetric. In order to give a local existence theorem, we will require $p_{q}$ to be small enough such that the system is, for small initial data, a strictly hyperbolic system.

Lemma 2.1. There exists $\delta$ such that if $|(\rho-1, \theta-1, q, S)|<\delta$, then the system (2.1) is strictly hyperbolic.

Proof. First, we choose a $\delta_{1}$ small enough such that $|(\rho-1, \theta-1, q, S)|<\delta_{1}$ implying

$$
\begin{array}{r}
0<\underline{\rho}<\rho<\bar{\rho}, 0<\underline{\theta}<\theta<\bar{\theta}, \\
0<\underline{p_{\rho}}<p_{\rho}<\overline{p_{\rho}}, 0<\underline{p_{\theta}}<p_{\theta}<\overline{p_{\theta}}, 0<\underline{e_{\theta}}<e_{\theta}<\overline{e_{\theta}},\left|p_{S}\right|<\frac{1}{2} .
\end{array}
$$

Now, we transform the system (2.1) into a first-order system for $V:=(\rho, u, \theta, q, S)^{\prime}$,

$$
V_{t}+A(V) \partial_{x} V+B(V) V=F(V),
$$

where

$$
A(V)=\left(\begin{array}{ccccc}
u & \rho & 0 & 0 & 0 \\
\frac{p_{\rho}}{\rho} & u & \frac{p_{\theta}}{\rho} & \frac{p_{q}}{\rho} & \frac{p_{S}-1}{\rho} \\
0 & \frac{\theta p_{\theta}}{\rho e_{\theta}} & u-\frac{2 q}{\rho \theta e_{\theta}} & 1 & 0 \\
0 & 0 & \frac{\kappa}{\tau_{1}} & u & 0 \\
0 & -\frac{\mu}{\tau_{2}} & 0 & 0 & u
\end{array}\right), B(V)=\left(\begin{array}{ccccc}
0 & 0 & 0 & 0 & 0 \\
0 & 0 & 0 & 0 & 0 \\
0 & 0 & 0 & 0 & 0 \\
0 & 0 & 0 & \frac{1}{\tau_{1}} & 0 \\
0 & 0 & 0 & 0 & \frac{1}{\tau_{2}}
\end{array}\right)
$$


and $F(V):=\left(0,0, \frac{2 q^{2}}{\kappa \theta}+\frac{S^{2}}{\mu}, 0,0\right)^{\prime}$. We need to show the eigenvalues of matrix $A(V)$ are real and distinct. The characteristic polynomial for $A(V)$ is

$$
\operatorname{det}(A(V)-\lambda)=(u-\lambda) g(u-\lambda),
$$

where

$$
\begin{aligned}
g(z):= & z^{4}-\frac{2 q}{\rho \theta e_{\theta}} z^{3}-\left(\frac{\kappa}{\tau_{1}}+\frac{\theta p_{\theta}^{2}}{\rho^{2} e_{\theta}}+\frac{\mu\left(1-p_{S}\right)}{\rho \tau_{2}}+p_{\rho}\right) z^{2} \\
& +\left(\frac{\kappa \theta p_{\theta} p_{q}}{\tau_{1} \rho^{2} e_{\theta}}+\left(\frac{\mu\left(1-p_{S}\right)}{\rho \tau_{2}}+p_{\rho}\right) \cdot \frac{2 q}{\rho \theta e_{\theta}}\right) z+\left(\frac{\mu\left(1-p_{S}\right)}{\rho \tau_{2}}+p_{\rho}\right) \cdot \frac{\kappa}{\tau_{1}} .
\end{aligned}
$$

Note that $g( \pm \infty)=+\infty$ and $g(0)=\left(\frac{\mu\left(1-p_{S}\right)}{\rho \tau_{2}}+p_{\rho}\right) \cdot \frac{\kappa}{\tau_{1}}>0$.

Let

$$
\mu_{ \pm}:= \pm \sqrt{\frac{\mu\left(1-p_{S}\right)}{\rho \tau_{2}}+p_{\rho}}
$$

then

$$
\mu_{-}<0<\mu_{+}
$$

and

$$
g\left(\mu_{ \pm}\right)=\mu_{-}\left(\frac{\theta p_{\theta}^{2}}{\rho^{2} e_{\theta}} \mu_{+} \mp \frac{\kappa \theta p_{\theta}}{\tau_{1} \rho^{2} e_{\theta}} p_{q}\right) \equiv \mu_{-} Q
$$

which implies

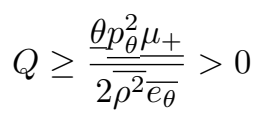

if

$$
\left|p_{q}\right| \leq \frac{\tau_{1} \underline{p_{\theta}}}{\kappa} \underline{\mu_{+}},
$$

which is satisfied if $|(v-1, \theta-1, q, S)|<\delta_{2}$ for some $\delta_{2}>0$. Therefore, there exists a $\delta_{2}>0$ such that for $|(\rho-1, \theta-1, q, S)|<\delta_{2}$, we derive

$$
g\left(\mu_{ \pm}\right)<0 .
$$

Hence, $g$ has 4 different real zeros $z_{1}<z_{2}<0<z_{3}<z_{4}$. Altogether we conclude that there exists a $\delta=\min \left\{\delta_{1}, \delta_{2}\right\}>0$ such that if $|(\rho-1, \theta-1, q, S)|_{L^{\infty}}<\delta$, the matrix $A(V)$ has 5 different eigenvalues $\lambda_{0}:=u, \lambda_{k}:=u-z_{k}, k=1,2,3,4$. Thus the system is strictly hyperbolic.

The strict hyperbolicity of (2.1) now implies the local well-posedness, see e.g. [34], it also implies that (2.1) is symmetrizable. Thus, we get

Theorem 2.2. Let $s \geq 2$. Then there is $\delta>0$ such that for $\left(\rho_{0}-1, u_{0}, \theta_{0}-1, q_{0}, S_{0}\right) \in W^{s, 2}(\mathbb{R})$ with $\left\|\left(\rho_{0}-1, u_{0}, \theta_{0}-1, q_{0}, S_{0}\right)\right\|_{s, 2}<\delta$, there exists a unique local solution $(\rho, u, \theta, q, S)$ to $(2.1)$, (1.4) in some time interval $[0, T]$ with

$$
(\rho-1, u, \theta-1, q, S) \in C^{0}\left([0, T], H^{s}(\mathbb{R})\right) \cap C^{1}\left([0, T], H^{s-1}(\mathbb{R})\right) .
$$




\section{Global well-Posedness: Proof of Theorem 1.1}

In this part, we establish the a priori estimates and prove the global existence of strong solutions by usual continuation methods. First, we introduce the following energy functional

$$
\begin{aligned}
E(t):=\sup _{0 \leq s \leq t} \|( & -1, u, \theta-1, q, S)(s, \cdot)\left\|_{H^{2}}^{2}+\sup _{0 \leq s \leq t}\right\|\left(\rho_{t}, u_{t}, \theta_{t}, q_{t}, S_{t}\right) \|_{H^{1}}^{2} \\
& +\int_{0}^{t}\left\|\left(\rho_{x}, \rho_{t}, u_{x}, u_{t}, \theta_{x}, \theta_{t}, q_{x}, q_{t}, q, S_{x}, S_{t}, S\right)(s, \cdot)\right\|_{H^{1}}^{2} \mathrm{~d} s .
\end{aligned}
$$

The following lemma gives the lower energy estimates.

Lemma 3.1. We have the following equality

$$
\begin{array}{r}
{\left[c_{v} \rho(\theta-\ln \theta-1)+R(\rho \ln \rho-\rho+1)+\left(1-\frac{1}{2 \theta}\right) \frac{\tau_{1}}{\kappa \theta} q^{2}+\frac{1}{2} \rho u^{2}+\frac{\tau_{2}}{2 \mu} S^{2}\right]_{t}} \\
+\left[\rho u c_{v}(\theta-\ln \theta-1)+u\left(1-\frac{1}{2 \theta}\right) \frac{\tau_{1}}{\kappa \theta} q^{2}+\frac{\tau_{2}}{2 \mu} u S^{2}+R \rho u \ln \rho-R \rho u-\frac{q}{\theta}+\frac{1}{2} \rho u^{3}+p u+q-S u\right]_{x} \\
+\frac{q^{2}}{\kappa \theta^{2}}+\frac{S^{2}}{\theta \mu}=0 .
\end{array}
$$

Moreover, if $E(t) \leq \frac{1}{16}$, we get the following $L^{2}$-energy estimate with a positive constant $C>0$,

$$
\int_{\mathbb{R}}\left((\rho-1)^{2}+u^{2}+(\theta-1)^{2}+q^{2}+S^{2}\right) \mathrm{d} x+\int_{0}^{t} \int_{\mathbb{R}}\left(q^{2}+S^{2}\right) \mathrm{d} x \mathrm{~d} t \leq C E(0) .
$$

Remark 3.1. When $\tau_{1}=\tau_{2}=0$, the equality (3.2) reduce to the energy inequality for classical compressible Navier-Stokes equaions, see [20]. In this regards, (3.2) can be regarded as an extension to the relaxed system (1.1)-(1.3). Moreover, if we denote, for $U=(\rho, u, \theta, q, S)$,

$$
\eta(U)=c_{v} \rho(\theta-\ln \theta-1)+R(\rho \ln \rho-\rho+1)+\left(1-\frac{1}{2 \theta}\right) \frac{\tau_{1}}{\kappa \theta} q^{2}+\frac{1}{2} \rho u^{2}+\frac{\tau_{2}}{2 \mu} S^{2},
$$

and

$q(U)=\rho u c_{v}(\theta-\ln \theta-1)+u\left(1-\frac{1}{2 \theta}\right) \frac{\tau_{1}}{\kappa \theta} q^{2}+\frac{\tau_{2}}{2 \mu} u S^{2}+R \rho u \ln \rho-R \rho u-\frac{q}{\theta}+\frac{1}{2} \rho u^{3}+p u+q-S u$,

we have $\eta(U)_{t}+q(U)_{x} \leq 0$ by (3.2). So, $(\eta(U), q(U))$ can be regards as a convex entropy pair for system (1.1)-(1.3). Then Lemma 3.1 imply that there exists a dissipative entropy which is crucial to get the global existence of solutions, see $[13,36]$.

Proof. First, we have the energy equality:

$$
\left(\rho e+\frac{1}{2} \rho u^{2}\right)_{t}+\left(\rho u e+\frac{1}{2} \rho u^{3}\right)_{x}+(p u)_{x}+q_{x}=(S u)_{x}
$$

which gives

$$
\int_{\mathbb{R}}\left(\rho e+\frac{1}{2} \rho u^{2}\right) \mathrm{d} x=\int_{\mathbb{R}}\left(\rho_{0} e_{0}+\frac{1}{2} \rho_{0} u_{0}^{2}\right) \mathrm{d} x
$$

or equivalently

$$
\int_{\mathbb{R}}\left(c_{v} \rho \theta+\frac{\tau_{1}}{\kappa \theta} q^{2}+\frac{\tau_{2}}{2 \mu} S^{2}+\frac{1}{2} \rho u^{2}\right) \mathrm{d} x=\int_{\mathbb{R}}\left(c_{v} \rho_{0} \theta_{0}+\frac{\tau_{1}}{\kappa \theta_{0}} q_{0}^{2}+\frac{\tau_{2}}{2 \mu} S_{0}^{2}+\frac{1}{2} \rho_{0} u_{0}^{2}\right) \mathrm{d} x .
$$


Next, we multiply the equation $(1.1)_{3}$ by $\frac{1}{\theta}$, we get

$$
\begin{aligned}
\frac{\rho}{\theta}\left(c_{v} \theta+\frac{\tau_{1}}{\kappa \theta \rho} q^{2}+\frac{\tau_{2}}{2 \mu \rho} S^{2}\right)_{t} & +\frac{\rho u}{\theta}\left(c_{v} \theta+\frac{\tau_{1}}{\kappa \theta \rho} q^{2}+\frac{\tau_{2}}{2 \mu \rho} S^{2}\right)_{x} \\
& +R \rho u_{x}-\frac{\tau_{1}}{2 \kappa \theta^{2}} q^{2} u_{x}-\frac{\tau_{2}}{2 \mu \theta} S^{2} u_{x}+\frac{q_{x}}{\theta}=\frac{S u_{x}}{\theta} .
\end{aligned}
$$

For the term $\frac{\rho}{\theta}\left(\frac{\tau_{1}}{\kappa \theta \rho} q^{2}\right)_{t}$, we have

$$
\begin{aligned}
\frac{\rho}{\theta}\left(\frac{\tau_{1}}{\kappa \theta \rho} q^{2}\right)_{t} & =\rho\left(\frac{\tau_{1}}{\kappa \rho \theta^{2}} q^{2}\right)_{t}+\frac{\tau_{1} \theta_{t}}{\kappa \theta^{3}} q^{2} \\
& =\rho\left(\frac{\tau_{1}}{\kappa \rho \theta^{2}} q^{2}\right)_{t}-\frac{1}{2}\left(\frac{1}{\theta^{2}}\right)_{t} \frac{\tau_{1}}{\kappa} q^{2} \\
& =\rho\left(\frac{\tau_{1}}{\kappa \rho \theta^{2}} q^{2}\right)_{t}-\frac{1}{2}\left(\frac{\tau_{1}}{\kappa \theta^{2}} q^{2}\right)_{t}+\frac{\tau_{1} q q_{t}}{\kappa \theta^{2}} \\
& =\rho\left(\frac{\tau_{1}}{\kappa \rho \theta^{2}} q^{2}\right)_{t}-\frac{1}{2}\left(\frac{\tau_{1}}{\kappa \theta^{2}} q^{2}\right)_{t}-u \frac{\tau_{1} q q_{x}}{\kappa \theta^{2}}-\frac{q^{2}}{\kappa \theta^{2}}-q \frac{\theta_{x}}{\theta^{2}} .
\end{aligned}
$$

For the term $\frac{\rho u}{\theta}\left(\frac{\tau_{1}}{\kappa \theta \rho} q^{2}\right)_{x}$, we get

$$
\begin{aligned}
\frac{\rho u}{\theta}\left(\frac{\tau_{1}}{\kappa \theta \rho} q^{2}\right)_{x} & =\rho u\left(\frac{\tau_{1}}{\kappa \rho \theta^{2}} q^{2}\right)_{x}+u \frac{\theta_{x}}{\theta^{3}} \frac{\tau_{1}}{\kappa} q^{2} \\
& =\rho u\left(\frac{\tau_{1}}{\kappa \rho \theta^{2}} q^{2}\right)_{x}-u\left(\frac{1}{2} \frac{\tau_{1}}{\kappa \theta^{2}} q^{2}\right)_{x}+u \frac{\tau_{1}}{\kappa \theta^{2}} q q_{x} .
\end{aligned}
$$

Then, we have

$$
\begin{aligned}
& \frac{\rho}{\theta}\left(\frac{\tau_{1}}{\kappa \theta \rho} q^{2}\right)_{t}+\frac{\rho u}{\theta}\left(\frac{\tau_{1}}{\kappa \rho} q^{2}\right)_{x}-\frac{\tau_{1}}{2 \kappa \theta^{2}} q^{2} u_{x} \\
& =\left[\rho\left(\frac{\tau_{1}}{\kappa \rho \theta^{2}} q^{2}\right)_{t}+\rho u\left(\frac{\tau_{1}}{\kappa \rho \theta^{2}} q^{2}\right)_{x}\right]-\left[\frac{1}{2}\left(\frac{\tau_{1}}{\kappa \theta^{2}} q^{2}\right)_{t}+\left(u \frac{1}{2} \frac{\tau_{1}}{\kappa \theta^{2}} q^{2}\right)_{x}\right]-\frac{q^{2}}{\kappa \theta^{2}}-q \frac{\theta_{x}}{\theta^{2}} \\
& =\left[\frac{1}{2}\left(\frac{\tau_{1}}{\kappa \theta^{2}} q^{2}\right)_{t}+\left(u \frac{1}{2} \frac{\tau_{1}}{\kappa \theta^{2}} q^{2}\right)_{x}\right]-\frac{q^{2}}{\kappa \theta^{2}}-q \frac{\theta_{x}}{\theta^{2}},
\end{aligned}
$$

where we used, exploiting $(1.1)_{1}$, the following fact

$$
\rho(f)_{t}+\rho u(f)_{x}=(\rho f)_{t}+(\rho u f)_{x}
$$

for any function $f$. On the other hand, we have

$$
\begin{aligned}
& \frac{\rho}{\theta}\left(\frac{\tau_{2}}{2 \mu \rho} S^{2}\right)_{t}+\frac{\rho u}{\theta}\left(\frac{\tau_{2}}{2 \mu \rho} S^{2}\right)_{x}-\frac{\tau_{2}}{2 \mu \theta} S^{2} u_{x} \\
& =\frac{\tau_{2}}{\theta \mu} S S_{t}-\frac{\tau_{2} S^{2}}{2 \mu \rho \theta} \rho_{t}+\frac{\tau_{2}}{\theta \mu} u S S_{x}-\frac{\tau_{2} S^{2}}{2 \mu \rho \theta} u \rho_{x}-\frac{\tau_{2}}{2 \mu \theta} S^{2} u_{x} \\
& =\frac{S}{\theta \mu}\left(\tau_{2}\left(S_{t}+u S_{x}\right)\right)-\frac{\tau_{2} S^{2}}{2 \mu \rho \theta}\left(\rho_{t}+u \rho_{x}\right)-\frac{\tau_{2}}{2 \mu \theta} S^{2} u_{x} \\
& =\frac{S}{\theta \mu}\left(-S+\mu u_{x}\right)+\frac{\tau_{2} S^{2}}{2 \mu \rho \theta} \rho u_{x}-\frac{\tau_{2}}{2 \mu \theta} S^{2} u_{x} \\
& =-\frac{S^{2}}{\theta \mu}+\frac{S}{\theta} u_{x} .
\end{aligned}
$$

Therefore, we derive the following equality

$$
\left(\rho c_{v} \ln \theta+\frac{\tau_{1}}{2 \kappa \theta^{2}} q^{2}\right)_{t}+\left(\rho u c_{v} \ln \theta+u \frac{\tau_{1}}{2 \kappa \theta^{2}} q^{2}\right)_{x}+R \rho u_{x}+\left(\frac{q}{\theta}\right)_{x}-\frac{q^{2}}{\kappa \theta^{2}}-\frac{S^{2}}{\theta \mu}=0 .
$$


Now, we rewrite the equation $(1.1)_{1}$ as

$$
R \rho(\ln \rho)_{t}+R \rho u(\ln \rho)_{x}+R \rho u_{x}=0 .
$$

Combining the equations (3.14), (3.15) and (3.6), using the equation $(1.1)_{1}$, we get

$$
\begin{array}{r}
{\left[c_{v} \rho(\theta-\ln \theta-1)+R(\rho \ln \rho-\rho+1)+\left(1-\frac{1}{2 \theta}\right) \frac{\tau_{1}}{\kappa \theta} q^{2}+\frac{1}{2} \rho u^{2}+\frac{\tau_{2}}{2 \mu} S^{2}\right]_{t}} \\
+\left[\rho u c_{v}(\theta-\ln \theta-1)+u\left(1-\frac{1}{2 \theta}\right) \frac{\tau_{1}}{\kappa \theta} q^{2}+\frac{\tau_{2}}{2 \mu} u S^{2}+R \rho u \ln \rho-R \rho u-\frac{q}{\theta}+\frac{1}{2} \rho u^{3}+p u+q-S u\right]_{x} \\
+\frac{q^{2}}{\kappa \theta^{2}}+\frac{S^{2}}{\theta \mu}=0 .
\end{array}
$$

Note that if $E(t) \leq \frac{1}{16}$, using Sobolev imbedding theorem, we have

$$
\frac{3}{4} \leq \rho \leq \frac{5}{4}, \quad \frac{3}{4} \leq \theta \leq \frac{5}{4} .
$$

Moreover, using Taylor expansions, we get

$$
\begin{aligned}
& \theta-\ln \theta-1=\frac{1}{2 \xi^{2}}(\theta-1)^{2}, \\
& \rho \ln \rho-\rho+1=\frac{1}{2 \eta}(\rho-1)^{2}
\end{aligned}
$$

where $\xi \in(1, \theta), \eta \in(1, \rho)$. Combining the above inequalities, we get the desired result.

The next two lemmas give the first-order estimates of solutions.

Lemma 3.2. Suppose that $E(t) \leq \delta$ for $0 \leq t \leq T$ and some sufficiently small $\delta>0$, then we have

$$
\begin{array}{r}
\int_{\mathbb{R}}\left(\rho_{x}^{2}+\rho_{t}^{2}+u_{x}^{2}+u_{t}^{2}+\theta_{x}^{2}+\theta_{t}^{2}+q_{x}^{2}+q_{t}^{2}+S_{x}^{2}+S_{t}^{2}\right) \mathrm{d} x(t) \\
+\int_{0}^{t} \int_{\mathbb{R}}\left(q_{x}^{2}+q_{t}^{2}+S_{x}^{2}+S_{t}^{2}\right) \mathrm{d} x \mathrm{~d} t \leq C\left(E(0)+E(t)^{\frac{3}{2}}\right)
\end{array}
$$

with a positive constant $C$.

Proof. Taking derivatives of (1.1) with respect to $\mathrm{x}$, we get

$$
\left\{\begin{array}{l}
\rho_{t x}+u \rho_{x x}+\rho u_{x x}=-2 u_{x} \rho_{x}=: f_{1}, \\
\rho u_{t x}+\rho u u_{x x}+p_{\rho} \rho_{x x}+p_{\theta} \theta_{x x}+p_{q} q_{x x}-\left(1-p_{S}\right) S_{x x} \\
=-\rho_{x} u_{t}-(\rho u)_{x} u_{x}-\left(p_{\rho}\right)_{x} \rho_{x}-\left(p_{\theta}\right)_{x} \theta_{x}-\left(p_{q}\right)_{x} q_{x}-\left(p_{S}\right)_{x} S_{x}=: f_{2}, \\
\rho e_{\theta} \theta_{t x}+\left(\rho u e_{\theta}-\frac{2 q}{\theta}\right) \theta_{x x}+\theta p_{\theta} u_{x x}+q_{x x} \\
=-\left(\rho e_{\theta}\right)_{x} \theta_{t}-\left(\rho u e_{\theta}-\frac{2 q}{\theta}\right)_{x} \theta_{x}-\left(\theta p_{\theta}\right)_{x} u_{x}+\left(\frac{2 q^{2}}{\kappa \theta}\right)_{x}+\left(\frac{S^{2}}{\mu}\right)_{x}=: f_{3}, \\
\tau_{1}\left(q_{t x}+u q_{x x}\right)+q_{x}+\kappa \theta_{x x}=-\tau_{1} u_{x} q_{x}=: f_{4}, \\
\tau_{2}\left(S_{t x}+u S_{x x}\right)+S_{x}-\mu u_{x x}=-\tau_{2} u_{x} S_{x}=: f_{5} .
\end{array}\right.
$$

Note that for $1 \leq j \leq 5$, there exists a constant $C$ such that

$$
\left|f_{j}\right| \leq C\left|\left(\rho_{x}, u_{x}, u_{t}, \theta_{x}, \theta_{t}, q_{x}, S_{x}, q, S\right)\right|^{2} .
$$


Multiplying the equations in (3.20) by $\frac{p_{\rho}}{\rho} \rho_{x}, u_{x}, \frac{1}{\theta} \theta_{x}, \frac{1}{\kappa \theta} q_{x}, \frac{1-p_{S}}{\mu} S_{x}$, respectively, and integrate the results over $\mathbb{R}$, we get

$$
\begin{aligned}
& \frac{\mathrm{d}}{\mathrm{d} t} \int_{\mathbb{R}}\left(\frac{p_{\rho}}{2 \rho} \rho_{x}^{2}+\frac{\rho}{2} u_{x}^{2}+\frac{\rho e_{\theta}}{2 \theta} \theta_{x}^{2}+\frac{\tau_{1}}{2 \kappa \theta} q_{x}^{2}+\frac{\tau_{2}\left(1-p_{S}\right)}{2 \mu} S_{x}^{2}\right) \mathrm{d} x \\
& +\int_{\mathbb{R}}\left(\frac{1}{\kappa \theta} q_{x}^{2}+\frac{1-p_{S}}{\mu} S_{x}^{2}\right) \mathrm{d} x+\int_{\mathbb{R}} p_{q} q_{x x} u_{x} \mathrm{~d} x \\
& =\int_{\mathbb{R}}\left(\frac{p_{\rho}}{\rho} \rho_{x} f_{1}+u_{x} f_{2}+\frac{\theta_{x}}{\theta} f_{3}+\frac{1}{\kappa \theta} q_{x} f_{4}+\frac{1-p_{S}}{\mu} S_{x} f_{5}\right) \mathrm{d} x \\
& +\int_{\mathbb{R}}\left(\frac{1}{2} \rho_{x}^{2}\left(\frac{p_{\rho}}{\rho}\right)_{t}+\frac{1}{2} u_{x}^{2} \rho_{t}+\frac{1}{2} \theta_{x}^{2}\left(\frac{\rho e_{\theta}}{\theta}\right)_{t}+\frac{1}{2} q_{x}^{2}\left(\frac{\tau_{1}}{\kappa \theta}\right)_{t}+\frac{1}{2} S_{x}^{2}\left(\frac{\tau_{2}\left(1-p_{S}\right)}{\mu}\right)_{t}\right) \mathrm{d} x \\
& +\int_{\mathbb{R}}\left(\left(\frac{p_{\rho}}{\rho} u\right)_{x} \frac{1}{2} \rho_{x}^{2}+(\rho u)_{x} \frac{1}{2} u_{x}^{2}+\left(\frac{\rho u e_{\theta}}{\theta}-\frac{2 q}{\theta^{2}}\right)_{x} \frac{1}{2} \theta_{x}^{2}+\left(\frac{u}{\kappa \theta}\right)_{x} \frac{\tau_{1}}{2} q_{x}^{2}+\left(\frac{1-p_{S}}{\mu} u\right)_{x} \frac{\tau_{2}}{2} S_{x}^{2}\right) \mathrm{d} x \\
& \left.+\int_{\mathbb{R}}\left(\left(p_{\rho}\right)\right)_{x} \rho_{x} u_{x}+\left(p_{\theta}\right)_{x} \theta_{x} u_{x}+\left(\frac{1}{\theta}\right)_{x} q_{x} \theta_{x}-\left(1-P_{S}\right)_{x} S_{x} u_{x}\right) .
\end{aligned}
$$

The integrals on the right-hand side of the above equation can be estimated by $E(t)^{\frac{3}{2}}$ by using the Sobolev embedding inequality

$$
\|(D \rho, D u, D \theta, D S, D q)\|_{L^{\infty}} \leq C E(t)^{\frac{1}{2}},
$$

where $D=\left(\partial_{t}, \partial_{x}\right)$.

Now, we deal with one of the highest-order terms, $\int_{\mathbb{R}} p_{q} q_{x x} u_{x} \mathrm{~d} x$, in the following way.

$$
\begin{aligned}
& \int_{\mathbb{R}} p_{q} q_{x x} u_{x} \mathrm{~d} x=-\int_{\mathbb{R}}\left(p_{q}\right)_{x} q_{x} u_{x} \mathrm{~d} x-\int_{\mathbb{R}} p_{q} q_{x} u_{x x} \mathrm{~d} x \\
& =-\int_{\mathbb{R}}\left(p_{q}\right)_{x} q_{x} u_{x} \mathrm{~d} x-\int_{\mathbb{R}} p_{q} q_{x}\left(-\frac{\rho e_{\theta}}{\theta p_{\theta}} \theta_{t x}-\frac{\rho u e_{\theta}-\frac{2 q}{\theta}}{\theta p_{\theta}} \theta_{x x}-\frac{1}{\theta p_{\theta}} q_{x x}+\frac{1}{\theta p_{\theta}} f_{3}\right) \mathrm{d} x \\
& =-\int_{\mathbb{R}}\left(p_{q}\right)_{x} q_{x} u_{x} \mathrm{~d} x-\int_{\mathbb{R}} \frac{p_{q}}{\theta p_{\theta}} q_{x} f_{3} \mathrm{~d} x-\int_{\mathbb{R}}\left(\frac{p_{q}}{\theta p_{\theta}}\right)_{x} \frac{1}{2} q_{x}^{2} \mathrm{~d} x \\
& +\int_{\mathbb{R}} \frac{p_{q} \rho e_{\theta}}{\theta p_{\theta}} q_{x} \theta_{t x} \mathrm{~d} x+\int_{\mathbb{R}} \frac{p_{q}\left(\rho u e_{\theta}-\frac{2 q}{\theta}\right)}{\theta p_{\theta}} q_{x} \theta_{x x} \mathrm{~d} x
\end{aligned}
$$

where we have used the temperature equation $(2.1)_{3}$, while, using the equation $(2.1)_{4}$ for $q$, we get for the second highest-order term

$$
\begin{aligned}
& \int_{\mathbb{R}} \frac{p_{q} \rho e_{\theta}}{\theta p_{\theta}} q_{x} \theta_{t x} \mathrm{~d} x \\
& =\frac{\mathrm{d}}{\mathrm{d} t} \int_{\mathbb{R}} \frac{p_{q} \rho e_{\theta}}{\theta p_{\theta}} q_{x} \theta_{x} \mathrm{~d} x-\int_{\mathbb{R}}\left(\frac{p_{q} \rho e_{\theta}}{\theta p_{\theta}}\right)_{t} q_{x} \theta_{x} \mathrm{~d} x-\int_{\mathbb{R}} \frac{p_{q} \rho e_{\theta}}{\theta p_{\theta}} q_{t x} \theta_{x} \mathrm{~d} x \\
& =\frac{\mathrm{d}}{\mathrm{d} t} \int_{\mathbb{R}} \frac{p_{q} \rho e_{\theta}}{\theta p_{\theta}} q_{x} \theta_{x} \mathrm{~d} x-\int_{\mathbb{R}}\left(\frac{p_{q} \rho e_{\theta}}{\theta p_{\theta}}\right)_{t} q_{x} \theta_{x} \mathrm{~d} x \\
& -\int_{\mathbb{R}} \frac{p_{q} \rho e_{\theta}}{\theta p_{\theta}}\left(\frac{1}{\tau_{1}} f_{4}-\frac{1}{\tau_{1}} q_{x}-\frac{\kappa}{\tau_{1}} \theta_{x x}-u_{x} q_{x}-u q_{x x}\right) \theta_{x} \mathrm{~d} x \\
& =\frac{\mathrm{d}}{\mathrm{d} t} \int_{\mathbb{R}} \frac{p_{q} \rho e_{\theta}}{\theta p_{\theta}} q_{x} \theta_{x} \mathrm{~d} x-\int_{\mathbb{R}} \frac{p_{q} \rho e_{\theta}}{\theta p_{\theta}} u q_{x} \theta_{x x}-\int_{\mathbb{R}}\left(\frac{p_{q} \rho e_{\theta}}{\theta p_{\theta}}\right)_{t} q_{x} \theta_{x} \mathrm{~d} x \\
& -\int_{\mathbb{R}}\left(\frac{p_{q} \rho e_{\theta}}{\theta p_{\theta}}\left(\frac{1}{\tau_{1}} f_{4}-\frac{1}{\tau_{1}} q_{x}\right) \theta_{x}+\left(\frac{p_{q} \rho e_{\theta}}{\theta p_{\theta}} u\right)_{x} q_{x} \theta_{x}-\left(\frac{\kappa p_{q} \rho e_{\theta}}{\tau_{1} \theta p_{\theta}}\right)_{x} \frac{1}{2} \theta_{x}^{2}\right) \mathrm{d} x .
\end{aligned}
$$


Therefore, we obtain

$$
\begin{aligned}
& \int_{\mathbb{R}} \frac{p_{q} \rho e_{\theta}}{\theta p_{\theta}} q_{x} \theta_{t x} \mathrm{~d} x+\frac{p_{q}\left(\rho u e_{\theta}-\frac{2 q}{\theta}\right)}{\theta p_{\theta}} q_{x} \theta_{x x} \mathrm{~d} x=\frac{\mathrm{d}}{\mathrm{d} t} \int_{\mathbb{R}} \frac{p_{q} \rho e_{\theta}}{\theta p_{\theta}} q_{x} \theta_{x} \mathrm{~d} x-\int_{\mathbb{R}} \frac{2 q p_{q}}{\theta^{2} p_{\theta}} q_{x} \theta_{x x} \\
& -\int_{\mathbb{R}}\left(\frac{p_{q} \rho e_{\theta}}{\theta p_{\theta}}\left(\frac{1}{\tau_{1}} f_{4}-\frac{1}{\tau_{1}} q_{x}\right) \theta_{x}+\left(\frac{p_{q} \rho e_{\theta}}{\theta p_{\theta}} u\right)_{x} q_{x} \theta_{x}-\left(\frac{\kappa p_{q} \rho e_{\theta}}{\tau_{1} \theta p_{\theta}}\right)_{x} \frac{1}{2} \theta_{x}^{2}+\left(\frac{p_{q} \rho e_{\theta}}{\theta p_{\theta}}\right)_{t} q_{x} \theta_{x}\right) \mathrm{d} x .
\end{aligned}
$$

For the term $\int_{\mathbb{R}} \frac{2 q p_{q}}{\theta^{2} p_{\theta}} q_{x} \theta_{x x} \mathrm{~d} x$, using the equation for $q$, we have

$$
\begin{aligned}
& \int_{\mathbb{R}} \frac{2 q p_{q}}{\theta^{2} p_{\theta}} q_{x} \theta_{x x} \mathrm{~d} x=\int_{\mathbb{R}} \frac{2 q p_{q}}{\theta^{2} p_{\theta}} q_{x}\left(\frac{-\tau_{1}}{\kappa}\left(q_{t x}+u q_{x x}+u_{x} q_{x}\right)-\frac{1}{\kappa} q_{x}\right) \mathrm{d} x \\
& =-\frac{\mathrm{d}}{\mathrm{d} t} \int_{\mathbb{R}} \frac{\tau_{1} q p_{q}}{\kappa \theta^{2} p_{\theta}} q_{x}^{2} \mathrm{~d} x+\int_{\mathbb{R}}\left(\frac{\tau_{1} q p_{q}}{\kappa \theta^{2} p_{\theta}}\right)_{t} q_{x}^{2} \mathrm{~d} x \\
& +\int_{\mathbb{R}}\left(\frac{2 q p_{q} \tau_{1}}{\theta^{2} p_{\theta} \kappa} u\right)_{x} \frac{1}{2} q_{x}^{2}-\int_{\mathbb{R}} \frac{2 q p_{q}}{\theta^{2} p_{\theta}} q_{x}^{2} \frac{\tau_{1}}{\kappa} u_{x} \mathrm{~d} x-\int_{\mathbb{R}} \frac{2 q p_{q}}{\kappa \theta^{2} p_{\theta}} q_{x}^{2} \mathrm{~d} x .
\end{aligned}
$$

Finally, we derive, integrating (3.22), that

$$
\begin{aligned}
\int_{\mathbb{R}}\left(\frac{p_{\rho}}{2 \rho} \rho_{x}^{2}+\frac{1}{2} \rho u_{x}^{2}+\frac{\rho e_{\theta}}{2 \theta} \theta_{x}^{2}+\right. & \left.\frac{\tau_{1}}{2 \kappa \theta} q_{x}^{2}+\frac{\tau_{1}\left(1-p_{S}\right)}{2 \mu} S_{x}^{2}+\frac{p_{q} \rho e_{\theta}}{\theta p_{\theta}} q_{x} \theta_{x}+\frac{\tau_{1} q p_{q}}{\kappa \theta^{2} p_{\theta}} q_{x}^{2}\right) \mathrm{d} x \\
& +\int_{0}^{t} \int_{\mathbb{R}} \frac{1}{\kappa \theta} q_{x}^{2}+\frac{1-p_{S}}{\mu} S_{x}^{2} \mathrm{~d} x \mathrm{~d} t \leq C\left(E(0)+E(t)^{\frac{3}{2}}\right)
\end{aligned}
$$

Note that

$$
p_{q}=-\frac{\tau_{1}}{\kappa \theta} q, e_{\theta}=c_{v}-\frac{\tau_{1}}{\kappa \theta^{2} \rho} q^{2}, p_{\theta}=R \rho+\frac{\tau_{1}}{2 \kappa \theta^{2}} q^{2}
$$

It is not difficult to see that for sufficiently small $\delta$, if $E(t) \leq \delta$, then we get from (3.28) the following estimate

$$
\int_{\mathbb{R}}\left(\rho_{x}^{2}+u_{x}^{2}+\theta_{x}^{2}+q_{x}^{2}+S_{x}^{2}\right) \mathrm{d} x+\int_{0}^{t} \int_{\mathbb{R}}\left(q_{x}^{2}+S_{x}^{2}\right) \mathrm{d} x \mathrm{~d} t \leq C\left(E(0)+E(t)^{\frac{3}{2}}\right) .
$$

Using similar methods, we obtain

$$
\int_{\mathbb{R}}\left(\rho_{t}^{2}+u_{t}^{2}+\theta_{t}^{2}+q_{t}^{2}+S_{t}^{2}\right) \mathrm{d} x+\int_{0}^{t} \int_{\mathbb{R}}\left(q_{t}^{2}+S_{t}^{2}\right) \mathrm{d} x \mathrm{~d} t \leq C\left(E(0)+E(t)^{\frac{3}{2}}\right) .
$$

Thfe estimates (3.29) and (3.30) prove (3.19).

Lemma 3.3. If $E(t) \leq \delta$ for $0 \leq t \leq T$ and some sufficiently small $\delta>0$, we have

$$
\int_{0}^{t} \int_{\mathbb{R}}\left(\rho_{t}^{2}+\rho_{x}^{2}+u_{t}^{2}+u_{x}^{2}+\theta_{t}^{2}+\theta_{x}^{2}\right) \mathrm{d} x \mathrm{~d} t \leq C\left(E(0)+E(t)^{\frac{3}{2}}\right) .
$$

Proof. Using Lemma 3.1 and Lemma 3.2 and the equations, we immediately get

$$
\int_{0}^{t} \int_{\mathbb{R}}\left(u_{x}^{2}+\theta_{x}^{2}+\theta_{t}^{2}\right) \mathrm{d} x \mathrm{~d} t \leq C\left(E(0)+E(t)^{\frac{3}{2}}\right) .
$$


On the other hand, multiplying the momentum equation $(1.1)_{2}$ by $u_{t}+u u_{x}$ and integrating over $(0, t) \times \mathbb{R}$, we get

$$
\begin{aligned}
& \int_{0}^{t} \int_{\mathbb{R}} \rho\left(u_{t}+u u_{x}\right)^{2} \mathrm{~d} x \mathrm{~d} t \\
& \leq-\int_{0}^{t} \int_{\mathbb{R}} R \theta \rho_{x}\left(u_{t}+u u_{x}\right) \mathrm{d} x \mathrm{~d} t+\frac{1}{4} \int_{0}^{t} \int_{\mathbb{R}} \rho\left(u_{t}+u u_{x}\right)^{2} \mathrm{~d} x \mathrm{~d} t+C\left(E(0)+E(t)^{\frac{3}{2}}\right) \\
& \leq \int_{0}^{t} \int_{\mathbb{R}}^{t} R \theta \rho u_{x t} \mathrm{~d} x \mathrm{~d} t-\int_{0}^{t} \int_{\mathbb{R}} R \theta \rho_{x} u u_{x} \mathrm{~d} x \mathrm{~d} t+\frac{1}{2} \int_{0}^{t} \int_{\mathbb{R}} \rho\left(u_{t}+u u_{x}\right)^{2} \mathrm{~d} x \mathrm{~d} t+C\left(E(0)+E(t)^{\frac{3}{2}}\right) \\
& \leq-\int_{0}^{t} \int_{\mathbb{R}} R \theta u_{x}\left(\rho_{t}+u \rho_{x}\right) \mathrm{d} x \mathrm{~d} t+\frac{1}{2} \int_{0}^{t} \int_{\mathbb{R}} \rho\left(u_{t}+u u_{x}\right)^{2} \mathrm{~d} x \mathrm{~d} t+C\left(E(0)+E(t)^{\frac{3}{2}}\right) \\
& \leq \frac{1}{2} \int_{0}^{t} \int_{\mathbb{R}} \rho\left(u_{t}+u u_{x}\right)^{2} \mathrm{~d} x \mathrm{~d} t+C\left(E(0)+E(t)^{\frac{3}{2}}\right)
\end{aligned}
$$

which together with (3.32) imply

$$
\int_{0}^{t} \int_{\mathbb{R}} u_{t}^{2} \mathrm{~d} x \mathrm{~d} t \leq C\left(E(0)+E(t)^{\frac{3}{2}}\right) .
$$

Now, using the equations (1.1) again, we get

$$
\int_{0}^{t} \int_{\mathbb{R}}\left(\rho_{t}^{2}+\rho_{x}^{2}\right) \mathrm{d} x \mathrm{~d} t \leq C\left(E(0)+E(t)^{\frac{3}{2}}\right)
$$

Next we give the second-order estimates of solutions.

Lemma 3.4. If $E(t) \leq \delta$ for $0 \leq t \leq T$ and some sufficiently $\delta>0$, we have

$$
\begin{aligned}
\int_{\mathbb{R}}\left(\rho_{x x}^{2}+\right. & \left.\rho_{t x}^{2}+u_{x x}^{2}+u_{t x}^{2}+\theta_{x x}^{2}+\theta_{t x}^{2}+q_{x x}^{2}+q_{x t}^{2}+S_{x x}^{2}+S_{t x}^{2}\right) \mathrm{d} x(t) \\
& +\int_{0}^{t} \int_{\mathbb{R}}\left(q_{x x}^{2}+q_{t x}^{2}+S_{x x}^{2}+S_{t x}^{2}\right) \mathrm{d} x \mathrm{~d} t \leq C\left(E(0)+E(t)^{\frac{3}{2}}\right) .
\end{aligned}
$$

Proof. In the following proof, we shall use higher-order derivatives, like $\rho_{t x x}, u_{x x x}$, to show the above estimate. Although these higher-order derivatives are not covered by Theorem 1.1, a standard density argument will eliminate the needs for the extra regularity of local solutions. (cf. the proof of Theorem 4.1 in [32]).

Taking derivatives of the equations(1.1) with respect to $\mathrm{x}$ twice, we get

$$
\begin{aligned}
& \rho_{t x x}+u \rho_{x x x}+\rho u_{x x x}=-u_{x} \rho_{x x}-\rho_{x} u_{x x}+\left(f_{1}\right)_{x}=: g_{1}, \\
& \rho u_{t x x}+\rho u u_{x x x}+p_{\rho} \rho_{x x x}+p_{\theta} \theta_{x x x}+p_{q} q_{x x x}-\left(1-p_{S}\right) S_{x x x} \\
& =-\rho_{x} u_{t x}-(\rho u)_{x} u_{x x}-\left(p_{\rho}\right)_{x} \rho_{x x}-\left(p_{\theta}\right)_{x} \theta_{x x}-\left(p_{q}\right)_{x} q_{x x}-\left(p_{S}\right)_{x} S_{x x}+\left(f_{2}\right)_{x}=: g_{2}, \\
& \rho e_{\theta} \theta_{t x x}+\left(\rho u e_{\theta}-\frac{2 q}{\theta}\right) \theta_{x x x}+\theta p_{\theta} u_{x x x}+q_{x x x} \\
& =-\left(\rho e_{\theta}\right)_{x} \theta_{t x}-\left(\rho u e_{\theta}-\frac{2 q}{\theta}\right)_{x} \theta_{x x}-\left(\theta p_{\theta}\right)_{x} u_{x x}+\left(f_{3}\right)_{x}=: g_{3}, \\
& \tau_{1}\left(q_{t x x}+u q_{x x x}\right)+q_{x x}+\kappa \theta_{x x x}=-\tau_{1} u_{x} q_{x x}+\left(f_{4}\right)_{x}=: g_{4}, \\
& \tau_{2}\left(S_{t x x}+u S_{x x x}\right)+S_{x x}-\mu u_{x x x}=-\tau_{2} u_{x} S_{x x}+\left(f_{5}\right)_{x}=: g_{5} .
\end{aligned}
$$

Note that for $1 \leq j \leq 5$, there exists a constant $C$ such that

$$
\left|g_{j}\right| \leq C\left|\left(\rho_{x x}, u_{x x}, u_{t x}, \theta_{x x}, \theta_{t x}, q_{x x}, S_{x x}, \rho_{x}, u_{x}, u_{t}, \theta_{x}, \theta_{t}, q_{x}, S_{x}, q, S\right)\right|^{2} .
$$


Multiplying the resulting equations by $\frac{p_{\rho}}{\rho} \rho_{x x}, u_{x x}, \frac{1}{\theta} \theta_{x x}, \frac{1}{\kappa \theta} q_{x x}, \frac{1-p_{S}}{\mu} S_{x x}$, respectively, and integrating the results over $\mathbb{R}$, we get

$\frac{\mathrm{d}}{\mathrm{d} t} \int_{\mathbb{R}}\left(\frac{p_{\rho}}{2 \rho} \rho_{x x}^{2}+\frac{\rho}{2} u_{x x}^{2}+\frac{\rho e_{\theta}}{2 \theta} \theta_{x x}^{2}+\frac{\tau_{1}}{2 \kappa \theta} q_{x x}^{2}+\frac{\tau_{2}\left(1-p_{S}\right)}{2 \mu} S_{x x}^{2}\right) \mathrm{d} x$

$+\int_{\mathbb{R}}\left(\frac{1}{\kappa \theta} q_{x x}^{2}+\frac{1-p_{S}}{\mu} S_{x x}^{2}\right) \mathrm{d} x+\int_{\mathbb{R}} p_{q} q_{x x x} u_{x x} \mathrm{~d} x$

$=\int_{\mathbb{R}}\left(\frac{p_{\rho}}{\rho} \rho_{x x} g_{1}+u_{x x} g_{2}+\frac{\theta_{x x}}{\theta} g_{3}+\frac{1}{\kappa \theta} q_{x x} g_{4}+\frac{1-p_{S}}{\mu} S_{x x} g_{5}\right) \mathrm{d} x$

$+\int_{\mathbb{R}}\left(\frac{1}{2} \rho_{x x}^{2}\left(\frac{p_{\rho}}{\rho}\right)_{t}+\frac{1}{2} u_{x x}^{2} \rho_{t}+\frac{1}{2} \theta_{x x}^{2}\left(\frac{\rho e_{\theta}}{\theta}\right)_{t}+\frac{1}{2} q_{x x}^{2}\left(\frac{\tau_{1}}{\kappa \theta}\right)_{t}+\frac{1}{2} S_{x x}^{2}\left(\frac{\tau_{2}\left(1-p_{S}\right)}{\mu}\right)_{t}\right) \mathrm{d} x$

$\int_{\mathbb{R}}\left(\left(\frac{p_{\rho}}{\rho} u\right)_{x} \frac{1}{2} \rho_{x x}^{2}+(\rho u)_{x} \frac{1}{2} u_{x x}^{2}+\left(\frac{\rho u e_{\theta}}{\theta}-\frac{2 q}{\theta^{2}}\right)_{x} \frac{1}{2} \theta_{x x}^{2}+\left(\frac{u}{\kappa \theta}\right)_{x} \frac{\tau_{1}}{2} q_{x x}^{2}+\left(\frac{1-p_{S}}{\mu} u\right)_{x} \frac{\tau_{2}}{2} S_{x x}^{2}\right) \mathrm{d} x$

$\left.+\int_{\mathbb{R}}\left(\left(p_{\rho}\right)\right)_{x} \rho_{x x} u_{x x}+\left(p_{\theta}\right)_{x} \theta_{x x} u_{x x}+\left(\frac{1}{\theta}\right)_{x} q_{x x} \theta_{x x}-\left(1-P_{S}\right)_{x} S_{x x} u_{x x}\right)$.

The integrals on the right-hand side can be estimated by $E(t)^{\frac{3}{2}}$ by using the Sobolev embedding inequality

$$
\|(D \rho, D u, D \theta, D S, D q)\|_{L^{\infty}}(t) \leq C E(t)^{\frac{1}{2}}
$$

where $D=\left(\partial_{t}, \partial_{x}\right)$. Using similar idea as in the proof of Lemma 3.2, we deal with the term $\int_{\mathbb{R}} p_{q} q_{x x x} u_{x x} \mathrm{~d} x$ as follows.

$$
\begin{aligned}
& \int_{\mathbb{R}} p_{q} q_{x x x} u_{x x} \mathrm{~d} x=-\int_{\mathbb{R}}\left(p_{q}\right)_{x} q_{x x} u_{x x} \mathrm{~d} x-\int_{\mathbb{R}} p_{q} q_{x x} u_{x x x} \mathrm{~d} x \\
& =-\int_{\mathbb{R}}\left(p_{q}\right)_{x} q_{x x} u_{x x} \mathrm{~d} x-\int_{\mathbb{R}} p_{q} q_{x x}\left(-\frac{\rho e_{\theta}}{\theta p_{\theta}} \theta_{t x x}-\frac{\rho u e_{\theta}-\frac{2 q}{\theta}}{\theta p_{\theta}} \theta_{x x x}-\frac{1}{\theta p_{\theta}} q_{x x x}+\frac{1}{\theta p_{\theta}} g_{3}\right) \mathrm{d} x \\
& =-\int_{\mathbb{R}}\left(p_{q}\right)_{x} q_{x x} u_{x x} \mathrm{~d} x-\int_{\mathbb{R}} \frac{p_{q}}{\theta p_{\theta}} q_{x x} g_{3} \mathrm{~d} x-\int_{\mathbb{R}}\left(\frac{p_{q}}{\theta p_{\theta}}\right)_{x} \frac{1}{2} q_{x x}^{2} \mathrm{~d} x \\
& +\int_{\mathbb{R}} \frac{p_{q} \rho e_{\theta}}{\theta p_{\theta}} q_{x x} \theta_{t x x} \mathrm{~d} x+\int_{\mathbb{R}} \frac{p_{q}\left(\rho u e_{\theta}-\frac{2 q}{\theta}\right)}{\theta p_{\theta}} q_{x x} \theta_{x x x} \mathrm{~d} x
\end{aligned}
$$

and

$$
\begin{aligned}
& \int_{\mathbb{R}} \frac{p_{q} \rho e_{\theta}}{\theta p_{\theta}} q_{x x} \theta_{t x x} \mathrm{~d} x \\
& =\frac{\mathrm{d}}{\mathrm{d} t} \int_{\mathbb{R}} \frac{p_{q} \rho e_{\theta}}{\theta p_{\theta}} q_{x x} \theta_{x x} \mathrm{~d} x-\int_{\mathbb{R}}\left(\frac{p_{q} \rho e_{\theta}}{\theta p_{\theta}}\right)_{t} q_{x x} \theta_{x x} \mathrm{~d} x-\int_{\mathbb{R}} \frac{p_{q} \rho e_{\theta}}{\theta p_{\theta}} q_{t x x} \theta_{x x} \mathrm{~d} x \\
& =\frac{\mathrm{d}}{\mathrm{d} t} \int_{\mathbb{R}} \frac{p_{q} \rho e_{\theta}}{\theta p_{\theta}} q_{x x} \theta_{x x} \mathrm{~d} x-\int_{\mathbb{R}}\left(\frac{p_{q} \rho e_{\theta}}{\theta p_{\theta}}\right)_{t} q_{x x} \theta_{x x} \mathrm{~d} x \\
& -\int_{\mathbb{R}} \frac{p_{q} \rho e_{\theta}}{\theta p_{\theta}}\left(\frac{1}{\tau_{1}} g_{4}-\frac{1}{\tau_{1}} q_{x x}-\frac{\kappa}{\tau_{1}} \theta_{x x x}-u q_{x x x}\right) \theta_{x x} \mathrm{~d} x \\
& =\frac{\mathrm{d}}{\mathrm{d} t} \int_{\mathbb{R}} \frac{p_{q} \rho e_{\theta}}{\theta p_{\theta}} q_{x x} \theta_{x x} \mathrm{~d} x-\int_{\mathbb{R}} \frac{p_{q} \rho e_{\theta}}{\theta p_{\theta}} u q_{x x} \theta_{x x x}-\int_{\mathbb{R}}\left(\frac{p_{q} \rho e_{\theta}}{\theta p_{\theta}}\right)_{t} q_{x x} \theta_{x x} \mathrm{~d} x \\
& -\int_{\mathbb{R}}\left(\frac{p_{q} \rho e_{\theta}}{\theta p_{\theta}}\left(\frac{1}{\tau_{1}} g_{4}-\frac{1}{\tau_{1}} q_{x x}\right) \theta_{x x}+\left(\frac{p_{q} \rho e_{\theta}}{\theta p_{\theta}} u\right)_{x} q_{x x} \theta_{x x}-\left(\frac{\kappa p_{q} \rho e_{\theta}}{\tau_{1} \theta p_{\theta}}\right)_{x} \frac{1}{2} \theta_{x x}^{2}\right) \mathrm{d} x .
\end{aligned}
$$


Therefore, we get

$$
\begin{aligned}
& \int_{\mathbb{R}} \frac{p_{q} \rho e_{\theta}}{\theta p_{\theta}} q_{x x} \theta_{t x x} \mathrm{~d} x+\frac{p_{q}\left(\rho u e_{\theta}-\frac{2 q}{\theta}\right)}{\theta p_{\theta}} q_{x x} \theta_{x x x} \mathrm{~d} x=\frac{\mathrm{d}}{\mathrm{d} t} \int_{\mathbb{R}} \frac{p_{q} \rho e_{\theta}}{\theta p_{\theta}} q_{x x} \theta_{x x} \mathrm{~d} x-\int_{\mathbb{R}} \frac{2 q p_{q}}{\theta^{2} p_{\theta}} q_{x x} \theta_{x x x} \\
& -\int_{\mathbb{R}}\left(\frac{p_{q} \rho e_{\theta}}{\theta p_{\theta}}\left(\frac{1}{\tau_{1}} g_{4}-\frac{1}{\tau_{1}} q_{x x}\right) \theta_{x x}+\left(\frac{p_{q} \rho e_{\theta}}{\theta p_{\theta}} u\right)_{x} q_{x x} \theta_{x x}-\left(\frac{\kappa p_{q} \rho e_{\theta}}{\tau_{1} \theta p_{\theta}}\right)_{x} \frac{1}{2} \theta_{x x}^{2}+\left(\frac{p_{q} \rho e_{\theta}}{\theta p_{\theta}}\right)_{t} q_{x x} \theta_{x x}\right) \mathrm{d} x .
\end{aligned}
$$

For the term $\int_{\mathbb{R}} \frac{2 q p_{q}}{\theta^{2} p_{\theta}} q_{x x} \theta_{x x x} \mathrm{~d} x$, using the equation for $q$, we have

$$
\begin{aligned}
& \int_{\mathbb{R}} \frac{2 q p_{q}}{\theta^{2} p_{\theta}} q_{x x} \theta_{x x x} \mathrm{~d} x=\int_{\mathbb{R}} \frac{2 q p_{q}}{\theta^{2} p_{\theta}} q_{x x}\left(\frac{-\tau_{1}}{\kappa}\left(q_{t x x}+u q_{x x x}\right)-\frac{1}{\kappa} q_{x x}+\frac{1}{\kappa} g_{4}\right) \mathrm{d} x \\
& =-\frac{\mathrm{d}}{\mathrm{d} t} \int_{\mathbb{R}} \frac{\tau_{1} q p_{q}}{\kappa \theta^{2} p_{\theta}} q_{x x}^{2} \mathrm{~d} x+\int_{\mathbb{R}}\left(\frac{\tau_{1} q p_{q}}{\kappa \theta^{2} p_{\theta}}\right)_{t} q_{x x}^{2} \mathrm{~d} x \\
& +\int_{\mathbb{R}}\left(\frac{2 q p_{q} \tau_{1}}{\theta^{2} p_{\theta} \kappa} u\right)_{x} \frac{1}{2} q_{x x}^{2}-\int_{\mathbb{R}} \frac{2 q p_{q}}{\kappa \theta^{2} p_{\theta}} q_{x x}^{2} \mathrm{~d} x+\int_{\mathbb{R}} \frac{2 q p_{q}}{\theta^{2} p_{\theta}} q_{x x} g_{4} .
\end{aligned}
$$

Finally, we derive that

$$
\begin{array}{r}
\int_{\mathbb{R}}\left(\frac{p_{\rho}}{2 \rho} \rho_{x x}^{2}+\frac{1}{2} \rho u_{x x}^{2}+\frac{\rho e_{\theta}}{2 \theta} \theta_{x x}^{2}+\frac{\tau_{1}}{2 \kappa \theta} q_{x x}^{2}+\frac{\tau_{1}\left(1-p_{S}\right)}{2 \mu} S_{x x}^{2}+\frac{p_{q} \rho e_{\theta}}{\theta p_{\theta}} q_{x x} \theta_{x x}+\frac{\tau_{1} q p_{q}}{\kappa \theta^{2} p_{\theta}} q_{x x}^{2}\right) \mathrm{d} x \\
+\int_{0}^{t} \int_{\mathbb{R}} \frac{1}{\kappa \theta} q_{x x}^{2}+\frac{1-p_{S}}{\mu} S_{x x}^{2} \mathrm{~d} x \mathrm{~d} t \leq C\left(E(0)+E(t)^{\frac{3}{2}}\right)
\end{array}
$$

Note that

$$
p_{q}=-\frac{\tau_{1}}{\kappa \theta} q, e_{\theta}=c_{v}-\frac{\tau_{1}}{\kappa \theta^{2} \rho} q^{2}, p_{\theta}=R \rho+\frac{\tau_{1}}{2 \kappa \theta^{2}} q^{2}
$$

So, there exists a small $\delta$ such that if $E(t) \leq \delta$, we can derive that

$$
\int_{\mathbb{R}}\left(\rho_{x x}^{2}+u_{x x}^{2}+\theta_{x x}^{2}+q_{x x}^{2}+S_{x x}^{2}\right) \mathrm{d} x+\int_{0}^{t} \int_{\mathbb{R}}\left(q_{x x}^{2}+S_{x x}^{2}\right) \mathrm{d} x \mathrm{~d} t \leq C\left(E(0)+E(t)^{\frac{3}{2}}\right) .
$$

Using similar methods, we can get

$$
\int_{\mathbb{R}}\left(\rho_{t x}^{2}+u_{t x}^{2}+\theta_{t x}^{2}+q_{t x}^{2}+S_{t x}^{2}\right) \mathrm{d} x+\int_{0}^{t} \int_{\mathbb{R}}\left(q_{t x}^{2}+S_{t x}^{2}\right) \mathrm{d} x \mathrm{~d} t \leq C\left(E(0)+E(t)^{\frac{3}{2}}\right) .
$$

Lemma 3.5. If $E(t) \leq \delta$ for $0 \leq t \leq T$ and some sufficiently small $\delta>0$, we have

$$
\int_{0}^{t} \int_{\mathbb{R}}\left(\rho_{t x}^{2}+\rho_{x x}^{2}+u_{t x}^{2}+u_{x x}^{2}+\theta_{t x}^{2}+\theta_{x x}^{2}\right) \mathrm{d} x \mathrm{~d} t \leq C\left(E(0)+E(t)^{\frac{3}{2}}\right) .
$$

Proof. Using Lemmas 3.1-3.4 and the system (3.20), we can immediately get

$$
\int_{0}^{t} \int_{\mathbb{R}}\left(u_{x x}^{2}+\theta_{x x}^{2}+\theta_{t x}^{2}\right) \mathrm{d} x \mathrm{~d} t \leq C\left(E(0)+E(t)^{\frac{3}{2}}\right) .
$$


On the other hand, multiplying the momentum equation $(3.20)_{2}$ by $u_{t x}+u u_{x x}$ and integrating over $(0, t) \times \mathbb{R}$, we get

$$
\begin{aligned}
& \int_{0}^{t} \int_{\mathbb{R}} \rho\left(u_{t x}+u u_{x x}\right)^{2} \mathrm{~d} x \mathrm{~d} t \\
& \leq-\int_{0}^{t} \int_{\mathbb{R}} R \theta \rho_{x x}\left(u_{t x}+u u_{x x}\right) \mathrm{d} x \mathrm{~d} t+\frac{1}{4} \int_{0}^{t} \int_{\mathbb{R}} \rho\left(u_{t x}+u u_{x x}\right)^{2} \mathrm{~d} x \mathrm{~d} t+C\left(E(0)+E(t)^{\frac{3}{2}}\right) \\
& \leq \int_{0}^{t} \int_{\mathbb{R}}^{t} R \theta \rho_{x} u_{t x x} \mathrm{~d} x \mathrm{~d} t-\int_{0}^{t} \int_{\mathbb{R}} R \theta \rho_{x x} u u_{x x} \mathrm{~d} x \mathrm{~d} t+\frac{1}{2} \int_{0}^{t} \int_{\mathbb{R}} \rho\left(u_{t x}+u u_{x x}\right)^{2} \mathrm{~d} x \mathrm{~d} t+C\left(E(0)+E(t)^{\frac{3}{2}}\right) \\
& \leq-\int_{0}^{t} \int_{\mathbb{R}} R \theta u_{x x}\left(\rho_{t x}+u \rho_{x x}\right) \mathrm{d} x \mathrm{~d} t+\frac{1}{2} \int_{0}^{t} \int_{\mathbb{R}} \rho\left(u_{t x}+u u_{x x}\right)^{2} \mathrm{~d} x \mathrm{~d} t+C\left(E(0)+E(t)^{\frac{3}{2}}\right) \\
& \leq \frac{1}{2} \int_{0}^{t} \int_{\mathbb{R}} \rho\left(u_{t x}+u u_{x x}\right)^{2} \mathrm{~d} x \mathrm{~d} t+C\left(E(0)+E(t)^{\frac{3}{2}}\right)
\end{aligned}
$$

which together with (3.53) imply

$$
\int_{0}^{t} \int_{\mathbb{R}} u_{t x}^{2} \mathrm{~d} x \mathrm{~d} t \leq C\left(E(0)+E(t)^{\frac{3}{2}}\right)
$$

Now, use the equations (3.20) again, we get

$$
\int_{0}^{t} \int_{\mathbb{R}}\left(\rho_{t x}^{2}+\rho_{x x}^{2}\right) \mathrm{d} x \mathrm{~d} t \leq C\left(E(0)+E(t)^{\frac{3}{2}}\right) .
$$

With the Lemmas 3.1-3.5 we have the necessary a priori estimates to continue, as usual for small data, the local solution from Theorem 2.2 to a global one. This proves Theorem 1.1.

\section{Relaxation limit}

In this section we prove Theorem 1.2, i.e. we show the uniform convergence of the relaxed system (1.1) to the classical compressible Navier-Stokes equations (corresponding to $\tau=\tau_{1}=\tau_{2}=0$ ).

Proof. Let $\rho^{d}=\frac{\rho^{\tau}-\rho}{\tau}, u^{d}=\frac{u^{\tau}-u}{\tau}, \theta^{d}=\frac{\theta^{\tau}-\theta}{\tau}, q^{d}=\frac{q^{\tau}-q}{\tau}, S^{d}=\frac{S^{\tau}-S}{\tau}$, where $q=-\kappa \theta_{x}$ and $S=\mu u_{x}$. It suffices to show that for small $\tau$ and $t<\min \left\{T_{*}, T_{\tau}\right\}$,

$$
\left\|\left(\rho^{d}, u^{d}, \theta^{d}\right)(t, \cdot)\right\|_{H^{2}} \leq C, \quad\left\|\sqrt{\tau}\left(q^{d}, S^{d}\right)(t, \cdot)\right\|_{H^{2}} \leq C,
$$

where $C>0$ will denote constants which do not depend on $\tau$.

We define

$$
E:=\sup _{0 \leq t \leq T}\left(\|(\rho-1, u, \theta-1)\|_{H^{5}}+\left\|\left(u_{t}, \theta_{t}\right)\right\|_{H^{3}}\right)
$$

and

$$
E^{d}:=\sup _{0 \leq t \leq T}\left\|\left(\rho^{d}, u^{d}, \theta^{d}, \sqrt{\tau} q^{d}, \sqrt{\tau} S^{d}\right)\right\|_{H^{2}} .
$$

Note that, by assumption, $E \leq C$ and

$$
\left\|\left(\rho^{\tau}-1, u^{\tau}, \theta^{\tau}-1\right)\right\|_{H^{2}} \leq C+\tau E^{d}, \quad\left\|\left(q^{\tau}, S^{\tau}\right)\right\|_{H^{2}} \leq C+\sqrt{\tau} E^{d} .
$$


The equations for the difference $\left(\rho^{d}, u^{d}, \theta^{d}, q^{d}, S^{d}\right)$ have the following form:

$$
\begin{aligned}
& \rho_{t}^{d}+u^{\tau} \rho_{x}^{d}+\rho^{\tau} u_{x}^{d}=-\rho^{d} u_{x}-u^{d} \rho_{x}=: f_{1}, \\
& u_{t}^{d}+u^{\tau} u_{x}^{d}+\frac{p_{\rho}^{\tau}}{\rho^{\tau}} \rho_{x}^{d}+\frac{p_{\theta}^{\tau}}{\rho^{\tau}} \theta_{x}^{d}+\frac{p_{q}^{\tau}}{\rho^{\tau}} q_{x}^{d}+\frac{\left(p_{S}^{\tau}-1\right)}{\rho^{\tau}} S_{x}^{d} \\
& =-\frac{\rho^{d} u_{t}}{\rho^{\tau}}-\frac{\rho^{\tau} u^{\tau}-\rho u}{\tau \rho^{\tau}} u_{x}-\frac{p_{\rho}^{\tau}-p_{\rho}}{\tau \rho^{\tau}} \rho_{x}-\frac{p_{\theta}^{\tau}-p_{\theta}}{\tau \rho^{\tau}} \theta_{x}-\frac{q_{x}}{\tau \rho^{\tau}} p_{q}^{\tau}-\frac{S_{x}}{\tau \rho^{\tau}} p_{S}^{\tau}=: f_{2}, \\
& \theta_{t}^{d}+\left(u^{\tau}-\frac{2 q^{\tau}}{\rho^{\tau} e_{\theta}^{\tau} \theta^{\tau}}\right) \theta_{x}^{d}+\frac{\theta^{\tau} p_{\theta}^{\tau}}{\rho^{\tau} e_{\theta}^{\tau}} u_{x}^{d}+\frac{1}{\rho^{\tau} e_{\theta}^{\tau}} q_{x}^{d} \\
& =-\frac{\rho^{\tau} e_{\theta}^{\tau}-\rho e_{\theta}}{\tau \rho^{\tau} e_{\theta}^{\tau}} \theta_{t}-\frac{\rho^{\tau} u^{\tau} e_{\theta}^{\tau}-\rho u e_{\theta}}{\tau \rho^{\tau} e_{\theta}^{\tau}} \theta_{x}-\frac{\theta^{\tau} p_{\theta}^{\tau}-\theta p_{\theta}}{\tau \rho^{\tau} e_{\theta}^{\tau}} u_{x}+\frac{2 q^{\tau}}{\kappa \theta^{\tau} \rho^{\tau} e_{\theta}^{\tau}} q^{d}+\frac{S^{\tau}+S}{\mu \rho^{\tau} e_{\theta}^{\tau}} S^{d}=: f_{3}, \\
& \tau\left(q_{t}^{d}+u^{\tau} q_{x}^{d}\right)+q^{d}+\kappa \theta_{x}^{d}=-\tau q_{x} u^{d}-\left(q_{t}+u q_{x}\right)=: f_{4}, \\
& \tau\left(S_{t}^{d}+u^{\tau} S_{x}^{d}\right)+S^{d}-\mu u_{x}^{d}=-\tau S_{x} u^{d}-\left(S_{t}+u S_{x}\right)=: f_{5} .
\end{aligned}
$$

Taking $\partial_{x}^{\alpha}(0 \leq \alpha \leq 2)$ to the above system, multiplying by $\frac{p_{\rho}^{\tau}}{\rho^{\tau}} \partial_{x}^{\alpha} \rho^{d}, \rho^{\tau} \partial_{x}^{\alpha} u^{d}, \frac{\rho^{\tau} e_{\theta}^{\tau}}{\theta^{\tau}} \partial_{x}^{\alpha} \theta^{d}, \frac{1}{\kappa \theta^{\tau}} \partial_{x}^{\alpha} q^{d}$ and $\frac{\left(1-p_{S}^{\tau}\right)}{\mu} \partial_{x}^{\alpha} S^{d}$, respectively, and integrating the result with respect to $x$, we obtain

$$
\begin{array}{r}
\frac{\mathrm{d}}{\mathrm{d} t} \int_{\mathbb{R}}\left(\frac{p_{\rho}^{\tau}}{2 \rho^{\tau}}\left(\partial_{x}^{\alpha} \rho^{d}\right)^{2}+\frac{\rho^{\tau}}{2}\left(\partial_{x}^{\alpha} u^{d}\right)^{2}+\frac{\rho^{\tau} e_{\theta}^{\tau}}{2 \theta^{\tau}}\left(\partial_{x}^{\alpha} \theta^{d}\right)^{2}+\frac{\tau}{2 \kappa \theta^{\tau}}\left(\partial_{x}^{\alpha} q^{d}\right)^{2}+\frac{\tau\left(1-p_{S}^{\tau}\right)}{2 \mu}\left(\partial_{x}^{\alpha} S^{d}\right)^{2}\right) \mathrm{d} x \\
+\int_{\mathbb{R}}\left(\frac{1}{\kappa \theta^{\tau}}\left(\partial_{x}^{\alpha} q^{d}\right)^{2}+\frac{\left(1-p_{S}^{\tau}\right)}{\mu}\left(\partial_{x}^{\alpha} S^{d}\right)^{2}\right) \mathrm{d} x+\int_{\mathbb{R}} \partial_{x}^{\alpha}\left(\frac{p_{q}^{\tau}}{\rho^{\tau}} q_{x}^{d}\right) \rho^{\tau} \partial_{x}^{\alpha} u^{d} \mathrm{~d} x \\
\equiv \sum_{i=1}^{4} T_{i}+\sum_{i=1}^{5} K_{i}+\sum_{i=1}^{4} M_{i}+\sum_{i=1}^{5} F_{i}
\end{array}
$$

where

$$
\begin{gathered}
T_{1}=\int_{\mathbb{R}} \frac{1}{2} \rho_{t}^{\tau}\left(\partial_{x}^{\alpha} u^{d}\right)^{2} \mathrm{~d} x, \quad T_{2}=\int_{\mathbb{R}} \frac{1}{2}\left(\frac{\rho^{\tau} e_{\theta}^{\tau}}{\theta^{\tau}}\right)_{t}\left(\partial_{x}^{\alpha} \theta^{d}\right)^{2} \mathrm{~d} x, \\
T_{3}=\int_{\mathbb{R}} \frac{\tau}{2}\left(\frac{1}{\kappa \theta^{\tau}}\right)_{t}\left(\partial_{x}^{\alpha} q^{d}\right)^{2} \mathrm{~d} x, \quad T_{4}=\int_{\mathbb{R}}\left(\frac{\tau\left(1-p_{S}^{\tau}\right)}{2 \mu}\right)_{t}\left(\partial_{x}^{\alpha} S^{d}\right)^{2} \mathrm{~d} x, \\
K_{1}=\int_{\mathbb{R}} \partial_{x}^{\alpha}\left(u^{\tau} \rho_{x}^{d}\right) \cdot \frac{p_{\rho}^{\tau}}{\rho^{\tau}} \partial_{x}^{\alpha} \rho^{d} \mathrm{~d} x, K_{2}=\int_{\mathbb{R}} \partial_{x}^{\alpha}\left(u^{\tau} u_{x}^{d}\right) \cdot\left(\rho^{\tau} \partial_{x}^{\alpha} u^{d}\right) \mathrm{d} x, \\
K_{3}=\int_{\mathbb{R}} \partial_{x}^{\alpha}\left(\left(u^{\tau}-\frac{2 q^{\tau}}{\rho^{\tau} e_{\theta}^{\tau} \theta^{\tau}}\right) \theta_{x}^{d}\right) \cdot \frac{\rho^{\tau} e_{\theta}^{\tau}}{\theta^{\tau}} \partial_{x}^{\alpha} \theta^{d} \mathrm{~d} x, \\
K_{4}=\int_{\mathbb{R}} \tau \partial_{x}^{\alpha}\left(u^{\tau} q_{x}^{d}\right) \cdot \frac{1}{\kappa \theta^{\tau}} \partial_{x}^{\alpha} q^{d} \mathrm{~d} x, \quad K_{5}=\int_{\mathbb{R}}^{\tau} \partial_{x}^{\alpha}\left(u^{\tau} S_{x}^{d}\right) \cdot \frac{1-p_{S}^{\tau}}{\mu} \partial_{x}^{\alpha} S^{d} \mathrm{~d} x, \\
M_{1}=\int_{\mathbb{R}}\left(\partial_{x}^{\alpha}\left(\rho^{\tau} u_{x}^{d}\right) \frac{p_{\rho}^{\tau}}{\rho^{\tau}} \partial_{x}^{\alpha} \rho^{d}+\partial_{x}^{\alpha}\left(\frac{p_{\rho}^{\tau}}{\rho^{\tau}} \rho_{x}^{d}\right) \rho^{\tau} \partial_{x}^{\alpha} u^{d}\right) \mathrm{d} x, \\
M_{2}=\int_{\mathbb{R}}\left(\partial_{x}\left(\frac{p_{\theta}^{\tau}}{\rho^{\tau}} \theta_{x}^{d}\right) \rho^{\tau} \partial_{x}^{\alpha} u^{d}+\partial_{x}^{\alpha}\left(\frac{\theta^{\tau} p_{\theta}^{\tau}}{\rho^{\tau} e_{\theta}^{\tau}} u_{x}^{d}\right) \frac{\rho^{\tau} e_{\theta}^{\tau}}{\theta^{\tau}} \partial_{x}^{\alpha} \theta^{d}\right) \mathrm{d} x, \\
M_{3}=\int_{\mathbb{R}}\left(\partial_{x}^{\alpha}\left(\frac{p_{S}^{\tau}-1}{\rho^{\tau}} S_{x}^{d}\right) \rho^{\tau} \partial_{x}^{\alpha} u^{d}-\mu \partial_{x}^{\alpha} u_{x}^{d} \frac{1-p_{S}^{\tau}}{\mu} \partial_{x}^{\alpha} S^{d}\right) \mathrm{d} x, \\
M_{4}=\int_{\mathbb{R}}\left(\partial_{x}^{\alpha}\left(\frac{1}{\rho^{\tau} e_{\theta}^{\tau}} q_{x}^{d}\right) \frac{\rho^{\tau} e_{\theta}^{\tau}}{\theta^{\tau}} \partial_{x}^{\alpha} \theta^{d}+\kappa \partial_{x}^{\alpha} \theta_{x}^{d} \frac{1}{\kappa \theta^{\tau}} \partial_{x}^{\alpha} q^{d}\right) \mathrm{d} x,
\end{gathered}
$$




$$
\begin{aligned}
& F_{1}=\int_{\mathbb{R}} \partial_{x}^{\alpha} f_{1} \cdot \frac{p_{\rho}^{\tau}}{\rho^{\tau}} \partial_{x}^{\alpha} \rho^{d} \mathrm{~d} x, F_{2}=\int_{\mathbb{R}} \partial_{x}^{\alpha} f_{2} \cdot \rho^{\tau} \partial_{x}^{\alpha} u^{\tau} \mathrm{d} x \\
& F_{3}=\int_{\mathbb{R}} \partial_{x}^{\alpha} f_{3} \cdot \frac{\rho^{\tau} e_{\theta}^{\tau}}{\theta^{\tau}} \partial_{x}^{\alpha} \theta^{d} \mathrm{~d} x, F_{4}=\int_{\mathbb{R}} \partial_{x}^{\alpha} f_{4} \cdot \frac{1}{\kappa \theta^{\tau}} \partial_{x}^{\alpha} q^{d} \mathrm{~d} x, F_{5}=\int_{\mathbb{R}} \partial_{x}^{\alpha} f_{5} \cdot \frac{1-p_{S}^{\tau}}{\mu} \partial_{x}^{\alpha} S^{d} \mathrm{~d} x .
\end{aligned}
$$

For $1 \leq i \leq 4$, we have

$$
\left|T_{i}\right| \leq C\left\|\left(\left(\rho^{\tau}\right)_{t},\left(\theta^{\tau}\right)_{t}, \tau\left(S^{\tau}\right)_{t}\right)\right\|_{L^{\infty}}\left(E^{d}\right)^{2} \leq C\left(\left(E^{d}\right)^{2}+\tau\left(E^{d}\right)^{3}+\tau^{2}\left(E^{d}\right)^{4}\right) .
$$

By using Moser type inequality and Sobolev imbedding theorem, we can get

$$
\begin{aligned}
& K_{1}=\int_{\mathbb{R}}\left(\partial_{x}^{\alpha}\left(u^{\tau} \rho_{x}^{d}\right)-u^{\tau} \partial_{x}^{\alpha+1} \rho^{d}\right) \cdot \frac{p_{\rho}^{\tau}}{\rho^{\tau}} \partial_{x}^{\alpha} \rho^{d} \mathrm{~d} x+\int_{\mathbb{R}} \frac{p_{\rho}^{\tau} u^{\tau}}{\rho^{\tau}} \partial_{x}^{\alpha+1} \rho^{d} \partial_{x}^{\alpha} \rho^{d} \\
& \leq C\left(\left\|\partial_{x}^{\alpha} u^{\tau}\right\|_{L^{2}}\left\|\rho_{x}^{d}\right\|_{L^{\infty}}+\left\|\partial_{x}^{\alpha} \rho^{d}\right\|_{L^{2}}\left\|\partial_{x} u^{\tau}\right\|_{L^{\infty}}\right) \cdot\left\|\frac{p_{\rho}^{\tau}}{\rho^{\tau}}\right\|_{L^{\infty}}\left\|\partial_{x}^{\alpha} \rho^{d}\right\|_{L^{2}}+\frac{1}{2}\left\|\left(\frac{p_{\rho}^{\tau} u^{\tau}}{\rho^{\tau}}\right)_{x}\right\|_{L^{\infty}}\left\|\partial_{x}^{\alpha} \rho^{d}\right\|_{L^{2}}^{2} \\
& \leq\left(C+\tau E^{d}\right)^{2} \cdot\left(E^{d}\right)^{2}+\left(C+\tau E^{d}\right)\left(E^{d}\right)^{2} \leq C\left(\left(E^{d}\right)^{2}+\tau\left(E^{d}\right)^{3}+\tau^{2}\left(E^{d}\right)^{4}\right) .
\end{aligned}
$$

Similarly, for $i=2,3,4$, we can get that

$$
\left|K_{i}\right| \leq C\left(\left(E^{d}\right)^{2}+\tau\left(E^{d}\right)^{3}+\tau^{2}\left(E^{d}\right)^{4}\right)
$$

We estimate $M_{1}$ as follows.

$$
\begin{aligned}
& M_{1}=\int_{\mathbb{R}}\left(\partial_{x}^{\alpha}\left(\rho^{\tau} u_{x}^{d}\right)-\rho^{\tau} \partial_{x}^{\alpha+1} u^{d}\right) \cdot \frac{p_{\rho}^{\tau}}{\rho^{\tau}} \partial_{x}^{\alpha} \rho^{d}+\left(\partial_{x}^{\alpha}\left(\frac{p_{\rho}^{\tau}}{\rho^{\tau}} \rho_{x}^{d}\right)-\frac{p_{\rho}^{\tau}}{\rho^{\tau}} \partial_{x}^{\alpha+1} \rho^{d}\right) \rho^{\tau} \partial_{x}^{\alpha} u^{d} \mathrm{~d} x \\
& \quad+\int_{\mathbb{R}} p_{\rho}^{\tau}\left(\partial_{x}^{\alpha+1} u^{d} \partial_{x}^{\alpha} \rho^{d}+\partial_{x}^{\alpha+1} \rho^{d} \partial_{x}^{\alpha} u^{d}\right) \mathrm{d} x \\
& \leq\left(C+\tau E^{d}\right)\left(\left\|\partial_{x} \rho^{\tau}\right\|_{L^{\infty}}\left\|\partial_{x}^{\alpha} u^{d}\right\|_{L^{2}}+\left\|u_{x}^{d}\right\|_{L^{\infty}}\left\|\partial_{x}^{\alpha} \rho^{\tau}\right\|_{L^{2}}\right)\left\|\partial_{x}^{\alpha} \rho^{d}\right\|_{L^{2}} \\
& \quad+\left(C+\tau E^{d}\right)\left(\left\|\partial_{x} \frac{p_{\rho}^{\tau}}{\rho^{\tau}}\right\|_{L^{\infty}}\left\|\partial_{x}^{\alpha} \rho^{d}\right\|+\left\|\rho_{x}^{d}\right\|_{L^{\infty}}\left\|\partial_{x}^{\alpha} \frac{p_{\rho}^{\tau}}{\rho^{\tau}}\right\|_{L^{2}}\right)\left\|\partial_{x}^{\alpha} u^{d}\right\|_{L^{2}}+\left\|\partial_{x} p_{\rho}^{\tau}\right\|_{L^{\infty}}\left\|\partial_{x}^{\alpha} u^{d}\right\|_{L^{2}}\left\|\partial_{x}^{\alpha} \rho^{d}\right\|_{L^{2}} \\
& \leq\left(C+\tau E^{d}\right)^{2} \cdot\left(E^{d}\right)^{2}+\left(C+\tau E^{d}\right)\left(E^{d}\right)^{2} \leq C\left(\left(E^{d}\right)^{2}+\tau\left(E^{d}\right)^{3}+\tau^{2}\left(E^{d}\right)^{4}\right) .
\end{aligned}
$$

Using similar methods, we can get also for $i=2,3,4$

$$
\left|M_{i}\right| \leq C\left(\left(E^{d}\right)^{2}+\tau\left(E^{d}\right)^{3}+\tau^{2}\left(E^{d}\right)^{4}\right) .
$$

Now we estimate the term $F_{i}$ for $i=1,2,3,4$, where the higher regularity assumptions on the solution are needed. First, we have

$$
\begin{aligned}
\left\|\partial_{x}^{\alpha} f_{1}\right\|_{L^{2}} & \leq\left\|\partial_{x}^{\alpha}\left(\rho^{d} u_{x}\right)\right\|_{L^{2}}+\left\|\partial_{x}^{\alpha}\left(u^{d} \rho_{x}\right)\right\|_{L^{2}} \\
& \leq\left\|\left(u_{x}, \rho_{x}\right)\right\|_{L^{\infty}}\left\|\left(\partial_{x}^{\alpha} \rho^{d}, \partial_{x}^{\alpha} u^{d}\right)\right\|_{L^{2}}+\left\|\left(\rho^{d}, u^{d}\right)\right\|_{L^{\infty}}\left\|\partial_{x}^{\alpha}\left(u_{x}, \rho_{x}\right)\right\|_{L^{2}} \leq C E^{d}, \\
\left\|\partial_{x}^{\alpha} f_{2}\right\|_{L^{2}} \leq & \left\|\partial_{x}^{\alpha}\left(\frac{\rho^{d} u_{t}}{\rho^{\tau}}\right)\right\|_{L^{2}}+\| \partial_{x}^{\alpha}\left(\frac { u _ { x } } { \rho ^ { \tau } } ( \rho ^ { \tau } u ^ { d } + u \rho ^ { d } ) \| _ { L ^ { 2 } } + \| \partial _ { x } ^ { \alpha } \left(\frac{p_{\rho}^{\tau}-p_{\rho}}{\rho^{\tau} \tau} \rho_{x} \|_{L^{2}}\right.\right. \\
& +\| \partial_{x}^{\alpha}\left(\frac{p_{\theta}^{\tau}-p_{\theta}}{\rho^{\tau} \tau} \theta_{x}\left\|_{L^{2}}+\right\| \partial_{x}^{\alpha}\left(\frac{p_{q}^{\tau}}{\rho^{\tau} \tau} q_{x}\right)\left\|_{L^{2}}+\right\| \partial_{x}^{\alpha}\left(\frac{p_{S}^{\tau}}{\rho^{\tau} \tau} S_{x}\right) \|_{L^{2}}\right. \\
\leq & C\left(\left\|\left(u_{t}, u_{x}, u u_{x}, \rho_{x}, \theta_{x}, q_{x}, S_{x}\right)\right\|_{\infty}\left\|\partial_{x}^{\alpha}\left(\frac{\rho^{d}}{\rho^{\tau}}, u^{d}, \frac{\rho^{d}}{\rho^{\tau}}, \frac{p_{\rho}^{\tau}-p_{\rho}}{\rho^{\tau} \tau}, \frac{p_{\theta}^{\tau}-p_{\theta}}{\rho^{\tau} \tau}, \frac{p_{q}^{\tau}}{\rho^{\tau} \tau}, \frac{p_{S}^{\tau}}{\rho^{\tau} \tau}\right)\right\|_{L^{2}}\right. \\
+ & \left.\left\|\partial_{x}^{\alpha}\left(u_{t}, u_{x}, u u_{x}, \rho_{x}, \theta_{x}, q_{x}, S_{x}\right)\right\|_{L^{2}}\left\|\left(\frac{\rho^{d}}{\rho^{\tau}}, u^{d}, \frac{\rho^{d}}{\rho^{\tau}}, \frac{p_{\rho}^{\tau}-p_{\rho}}{\rho^{\tau} \tau}, \frac{p_{\theta}^{\tau}-p_{\theta}}{\rho^{\tau} \tau}, \frac{p_{q}^{\tau}}{\rho^{\tau} \tau}, \frac{p_{S}^{\tau}}{\rho^{\tau} \tau}\right)\right\|_{\infty}\right) \\
\leq & C E^{d}\left(C+\tau E^{d}\right)
\end{aligned}
$$


where we have used the fact that $\frac{p_{q}^{\tau}}{\tau}=-\frac{q^{\tau}}{\kappa \theta^{\tau}}$ and $\frac{p_{S}^{\tau}}{\tau}=-\frac{S^{\tau}}{\mu}$. Similarly, we can get

$$
\begin{aligned}
& \left\|\partial_{x}^{\alpha} f_{3}\right\|_{L^{2}} \leq\left\|\left(\theta_{t}, \theta_{x}, S\right)\right\|_{L^{\infty}}\left\|\partial_{x}^{\alpha}\left(\frac{\rho^{\tau} e_{\theta}^{\tau}-\rho e_{\theta}}{\tau \rho^{\tau} e_{\theta}^{\tau}}, \frac{\rho^{\tau} u^{\tau} e_{\theta}^{\tau}-\rho u e_{\theta}}{\rho^{\tau} \tau}, \frac{S^{d}}{\mu \rho^{\tau} e_{\theta}^{\tau}}\right)\right\|_{L^{2}} \\
& \quad+\left\|\partial_{x}^{\alpha}\left(\theta_{t}, \theta_{x}, S\right)\right\|_{L^{2}}\left\|\left(\frac{\rho^{\tau} e_{\theta}^{\tau}-\rho e_{\theta}}{\tau \rho^{\tau} e_{\theta}^{\tau}}, \frac{\rho^{\tau} u^{\tau} e_{\theta}^{\tau}-\rho u e_{\theta}}{\rho^{\tau} \tau}, \frac{S^{d}}{\mu \rho^{\tau} e_{\theta}^{\tau}}\right)\right\|_{L^{\infty}} \\
& +\left\|\left(\frac{2 q^{\tau}}{\kappa \theta^{\tau} \rho^{\tau} e_{\theta}^{\tau}}, \frac{S^{\tau}}{\mu \rho^{\tau} e_{\theta}^{\tau}}\right)\right\|_{L^{\infty}}\left\|\partial_{x}^{\alpha}\left(q^{d}, S^{d}\right)\right\|_{L^{2}}+\left\|\partial_{x}^{\alpha}\left(\frac{2 q^{\tau}}{\kappa \theta^{\tau} \rho^{\tau} e_{\theta}^{\tau}}, \frac{S^{\tau}}{\mu \rho^{\tau} e_{\theta}^{\tau}}\right)\right\|_{L^{2}}\left\|\left(q^{d}, S^{d}\right)\right\|_{\infty} \\
& \leq C\left(E^{d}+\tau\left(E^{d}\right)^{2}+\tau^{2}\left(E^{d}\right)^{3}\right) .
\end{aligned}
$$

Note that $q=-\kappa \theta_{x}, S=\mu u_{x}$, so we get

$$
\|\left(\partial_{x}^{\alpha}\left(f_{4}, f_{5}\right) \|_{L^{2}} \leq C+C \tau E^{d} .\right.
$$

Based on the above estimates, we conclude

$$
\sum_{i=1}^{4} T_{i}+\sum_{i=1}^{5} K_{i}+\sum_{i=1}^{4} M_{i}+\sum_{i=1}^{4} F_{i} \leq C\left(1+\left(E^{d}\right)^{2}+\tau\left(E^{d}\right)^{3}+\tau^{2}\left(E^{d}\right)^{4}\right) .
$$

Finally, we deal with the last term on the left-hand side of (4.2), the term $\int_{\mathbb{R}} \partial_{x}^{\alpha}\left(\frac{p_{q}^{\tau}}{\rho^{\tau}} q_{x}^{d}\right) \rho^{\tau} \partial_{x}^{\alpha} u^{d} \mathrm{~d} x$.

$$
\begin{aligned}
& \int_{\mathbb{R}} \partial_{x}^{\alpha}\left(\frac{p_{q}^{\tau}}{\rho^{\tau}} q_{x}^{d}\right) \rho^{\tau} \partial_{x}^{\alpha} u^{d} \mathrm{~d} x \\
& =\int_{\mathbb{R}}\left(\partial_{x}^{\alpha}\left(\frac{p_{q}^{\tau}}{\rho^{\tau}} q_{x}^{d}\right)-\frac{p_{q}^{\tau}}{\rho^{\tau}} \partial_{x}^{\alpha+1} q^{d}\right) \rho^{\tau} \partial_{x}^{\alpha} u^{d} \mathrm{~d} x+\int_{\mathbb{R}} p_{q}^{\tau} \partial_{x}^{\alpha+1} q^{d} \partial_{x}^{\alpha} u^{d} \mathrm{~d} x \equiv I_{1}+I_{2} .
\end{aligned}
$$

Using Moser type inequality and the Sobolev imbedding theorem, we get

$$
I_{1} \leq C\left(\left\|\partial_{x}\left(\frac{p_{q}^{\tau}}{\rho^{\tau}}\right)\right\|_{L^{\infty}}\left\|\partial_{x}^{\alpha} q^{d}\right\|_{L^{2}}+\left\|q_{x}^{d}\right\|_{\infty}\left\|\partial_{x}^{\alpha} \frac{p_{q}^{\tau}}{\rho^{\tau}}\right\|_{L^{2}}\right)\left\|\partial_{x}^{\alpha} u^{d}\right\|_{L^{2}} \leq C\left(\left(E^{d}\right)^{2}+\tau\left(E^{d}\right)^{3}\right) .
$$

For the term $I_{2}$, we use similar methods as in the proof of Lemma 3.2.

$$
\begin{aligned}
& I_{2}=-\int_{\mathbb{R}}\left(p_{q}^{\tau}\right)_{x} \partial_{x}^{\alpha} q^{d} \partial_{x}^{\alpha} u^{d} \mathrm{~d} x-\int_{\mathbb{R}} p_{q}^{\tau} \partial_{x}^{\alpha} q^{d} \partial_{x}^{\alpha+1} u^{d} \mathrm{~d} x \\
& \leq \int_{\mathbb{R}} \frac{p_{q}^{\tau} \rho^{\tau} e_{\theta}^{\tau}}{\theta^{\tau} p_{\theta}^{\tau}} \partial_{x}^{\alpha} q^{d} \partial_{x}^{\alpha} \partial_{t} \theta^{d} \mathrm{~d} x+\int_{\mathbb{R}} \frac{p_{q}^{\tau} \rho^{\tau} e_{\theta}^{\tau}}{\theta^{\tau} p_{\theta}^{\tau}}\left(u^{\tau}-\frac{2 q^{\tau}}{\rho^{\tau} e_{\theta}^{\tau} \theta^{\tau}}\right) \partial_{x}^{\alpha} q^{d} \partial_{x}^{\alpha+1} \theta \mathrm{d} x \\
& \quad+C\left(\left(E^{d}\right)^{2}+\tau\left(E^{d}\right)^{3}+\tau^{2}\left(E^{d}\right)^{4}+\tau^{3}\left(E^{d}\right)^{5}\right) \\
& \leq \frac{\mathrm{d}}{\mathrm{d} t} \int_{\mathbb{R}} \frac{p_{q}^{\tau} \rho^{\tau} e_{\theta}^{\tau}}{\theta^{\tau} p_{\theta}^{\tau}} \partial_{x}^{\alpha} q^{d} \partial_{x}^{\alpha} \theta^{d} \mathrm{~d} x-\int_{\mathbb{R}} \frac{p_{q}^{\tau} \rho^{\tau} e_{\theta}^{\tau}}{\theta^{\tau} p_{\theta}^{\tau}} \partial_{x}^{\alpha} \partial_{t} q^{d} \partial_{x}^{\alpha} \theta^{d} \mathrm{~d} x+\int_{\mathbb{R}} \frac{p_{q}^{\tau} \rho^{\tau} e_{\theta}^{\tau}}{\theta^{\tau} p_{\theta}^{\tau}}\left(u^{\tau}-\frac{2 q^{\tau}}{\rho^{\tau} e_{\theta}^{\tau} \theta^{\tau}}\right) \partial_{x}^{\alpha} q^{d} \partial_{x}^{\alpha+1} \theta \mathrm{d} x \\
& \quad+C\left(\left(E^{d}\right)^{2}+\tau\left(E^{d}\right)^{3}+\tau^{2}\left(E^{d}\right)^{4}+\tau^{3}\left(E^{d}\right)^{5}\right) \\
& \leq \frac{\mathrm{d}}{\mathrm{d} t} \int_{\mathbb{R}} \frac{p_{q}^{\tau} \rho^{\tau} e_{\theta}^{\tau}}{\theta^{\tau} p_{\theta}^{\tau}} \partial_{x}^{\alpha} q^{d} \partial_{x}^{\alpha} \theta^{d} \mathrm{~d} x-\int_{\mathbb{R}} \frac{2 p_{q}^{\tau} q^{\tau}}{\left(\theta^{\tau}\right)^{2} p_{\theta}^{\tau}} \partial_{x}^{\alpha} q^{d} \partial_{x}^{\alpha+1} \theta \mathrm{d} x+C\left(\left(E^{d}\right)^{2}+\tau\left(E^{d}\right)^{3}+\tau^{2}\left(E^{d}\right)^{4}+\tau^{3}\left(E^{d}\right)^{5}\right) \\
& \leq \frac{\mathrm{d}}{\mathrm{d} t} \int_{\mathbb{R}}\left(\frac{p_{q}^{\tau} \rho^{\tau} e_{\theta}^{\tau}}{\theta^{\tau} p_{\theta}^{\tau}} \partial_{x}^{\alpha} q^{d} \partial_{x}^{\alpha} \theta^{d}+\frac{\tau p_{q}^{\tau} q^{\tau}}{\kappa\left(\theta^{\tau}\right)^{2} p_{\theta}^{\tau}}\left(\partial_{x}^{\alpha} q^{d}\right)^{2}\right) \mathrm{d} x+C\left(\left(E^{d}\right)^{2}+\tau\left(E^{d}\right)^{3}+\tau^{2}\left(E^{d}\right)^{4}+\tau^{3}\left(E^{d}\right)^{5}\right) .
\end{aligned}
$$


Combining (4.2)-(4.6), we finally derive that

$$
\begin{array}{r}
\frac{\mathrm{d}}{\mathrm{d} t} \int_{\mathbb{R}}\left(\frac{1}{2}\left(\partial_{x}^{\alpha} \rho^{d}\right)^{2}+\frac{\rho^{\tau}}{2}\left(\partial_{x}^{\alpha} u^{d}\right)^{2}+\frac{\rho^{\tau} e_{\theta}^{\tau}}{2 \theta^{\tau}}\left(\partial_{x}^{\alpha} \theta^{d}\right)^{2}+\frac{\tau}{2 \kappa \theta^{\tau}}\left(\partial_{x}^{\alpha} q^{d}\right)^{2}+\frac{\tau\left(1-p_{S}^{\tau}\right)}{2 \mu}\left(\partial_{x}^{\alpha} S^{d}\right)^{2}\right. \\
\left.+\frac{p_{q}^{\tau} \rho^{\tau} e_{\theta}^{\tau}}{\theta^{\tau} p_{\theta}^{\tau}} \partial_{x}^{\alpha} q^{d} \partial_{x}^{\alpha} \theta^{d}+\frac{\tau p_{q}^{\tau} q^{\tau}}{\kappa\left(\theta^{\tau}\right)^{2} p_{\theta}^{\tau}}\left(\partial_{x}^{\alpha} q^{d}\right)^{2}\right) \mathrm{d} x \\
+\int_{\mathbb{R}}\left(\frac{1}{\kappa \theta^{\tau}}\left(\partial_{x}^{\alpha} q^{d}\right)^{2}+\frac{\left(1-p_{S}^{\tau}\right.}{\mu}\left(\partial_{x}^{\alpha} S^{d}\right)^{2}\right) \mathrm{d} x \leq C\left(1+\left(E^{d}\right)^{2}+\tau\left(E^{d}\right)^{3}+\tau^{2}\left(E^{d}\right)^{4}+\tau^{3}\left(E^{d}\right)^{5}\right) .
\end{array}
$$

Summing $\alpha$ from 0 to 2 and noting that $p_{q}$ is sufficiently small if we choose $\tau$ sufficiently small, we get

$$
\frac{\mathrm{d}}{\mathrm{d} t}\left(E^{d}\right)^{2} \leq C\left(1+\left(E^{d}\right)^{2}+\tau\left(E^{d}\right)^{3}+\tau^{2}\left(E^{d}\right)^{4}+\tau^{3}\left(E^{d}\right)^{5}\right) .
$$

Thus, use the same arguments as in $[15,16]$, we conclude that there is a small $\tau_{0}>0$ such that for $0<\tau<\tau_{0}$,

$$
E^{d} \leq C
$$

This proves (4.1) and hence Theorem 1.2.

Acknowledgement: Yuxi Hu's research is supported by NNSFC (Grant No. 11701556, 11601385).

\section{REFERENCES}

[1] D. Chakraborty and J.E. Sader, Constitutive models for linear compressible viscoelastic flows of simple liquids at nanometer length scales, Physics of Fluids 27 (2015), 052002-1-052002-13.

[2] P.J. Chen and M.E. Gurtin, On second sound in materials with memory, Z. Ang. Math. Phys. 21 (1970), 232-241.

[3] Y. Cho and B.J. Jin, Blow-up of viscous heat-conducting compressible flows, J. Math. Anal. Appl. 320 (2) (2006), 819-826.

[4] H.J. Choe and H. Kim, Strong solutions of the Navier-Stokes equations for isentropic compressible fluids, J. Differ. Eqs. 190 (2003), 504-523.

[5] C.I. Christov and P.M. Jordan, Heat condction paradox involving second-sound propagation in moving media, Phys. Rev. Letters 94 (2005), 154301-1-154301-4.

[6] B.D. Coleman, M. Fabrizio and D.R. Owen, On the thermodynamics of second sound in dielectric crystals, Arch. Rational Mech. Anal. 80 (1986), 135-158.

[7] B.D. Coleman, W.J. Hrusa and D.R. Owen, Stability of Equilibrium for a Nonlinear Hyperbolic System Describing Heat Propagation by Second Sound in Solids, Arch. Rational Mech. Anal. 94 (1986), 267-289.

[8] H.D. Fernández Sare and J.E. Muñoz Rivera, Optimal rates of decay in 2-d thermoelasticity with second sound, J. Math. Phys. 53 (2012), 073509.

[9] H.D. Fernández Sare and R. Racke, On the stability of damped Timoshenko systems - Cattaneo versus Fourier law. Arch. Rational Mech. Anal. 194 (2009), 221-251.

[10] D. Hoff, Global existence for 1D, compressible, isentropic Navier-Stokes equations with large initial data, Trans. Amer. Math. Soc. 303 (1) (1987), 169-181.

[11] D. Hoff, Global solutions of the Navier-Stokes equations for multidimensional compressible flow with discontinuous initial data, J. Differ. Eqs. 120 (1) (1995), 215-254.

[12] E. Feireisl, A. Novotny and H. Petzeltová, On the existence of globally defined weak solutions to the NavierStokes equations, J. Math. Fluid Mech. 3 (2001), 358-392.

[13] B. Hanouzet and R. Natalini, Global existence of smooth solutions for partially dissipative hyperbolic systems with a convex entropy, Arch. Rational Mech. Anal. 169 (2003), 89-117.

[14] Y. Hu and N. Wang, Global existence versus blow-up results for one dimensional compressible Navier-Stokes equations with Maxwell's law, Math. Nachr. 292 (2019), 826-840.

[15] Y. Hu and R. Racke, Compressible Navier-Stokes equations with hyperbolic heat conduction, J. Hyper. Diff. Equ. 13 (2) (2016), 233-247.

[16] Y. Hu and R. Racke, Compressible Navier-Stokes equations with revised Maxwell's law, J. Math.Fluid Mech. 19 (2017), 77-90. 
[17] X.D. Huang, J. Li and Z.P. Xin, Global well-posedness of classical solutions with large oscillations and vacuum to the three-dimensional isentropic compressible Navier-Stokes equations, Comm. Pure. Appl. Math. 65 (2012), 549-585.

[18] S. Jiang and P. Zhang, Global spherically symmetry solutions of the compressible isentropic Navier-Stokes equations, Comm. Math. Phys. 215 (2001), 559-581.

[19] S. Jiang and P. Zhang, Axisymmetric solutions of the 3-D Navier-Stokes equations for compressible isentropic fluids, J. Math. Pures. Appl. 82 (2003), 949-973.

[20] A.V. Kazhikhov, Cauchy problem for viscous gas equations, Siberian Mathematical Journal 23 (1982), 44-49.

[21] P.L. Lions, Mathematical Topics in Fluid Mechanics, Vol.I, Incompressible Models. Clarendon Press, Oxford (1996).

[22] P.L. Lions, Mathematical Topics in Fluid Mechanics, Vol.II, Compressible Models. Clarendon Press, Oxford (1998).

[23] A. Matsumura and T. Nishida, The initial value problem for the equations of motion of viscous and heatconductive gases, J. Math. Kyoto Univ. 20 (1) (1980), 67-104.

[24] A. Matsumura and T. Nishida, Initial Boundary Value Problems for the Equations of Motion of Compressible Viscous and Heat-Conductive Fluids, Comm. Math. Phys. 89 (1983), 445-464.

[25] J. Nash, Le problème de Cauchy pour les équations différentielles d'un fluide général, Bull. Soc. Math. France 90 (1962), 487-497.

[26] R. Quintanilla and R. Racke, Addendum to: Qualitative aspects of solutions in resonators, Arch. Mech. 63 (2011), 429-435.

[27] R. Racke and J. Saal, Hyperbolic Navier-Stokes equations I: Local well-posedness, Evolution Equations and Control Theory 1 (2012), 195-215.

[28] R. Racke and J. Saal. Hyperbolic Navier-Stokes equations II: Global existence of small solutions, Evolution Equations and Control Theory 1 (2012), 217-234.

[29] A. Schöwe, A quasilinear delayed hyperbolic Navier-Stokes system: global solution, asymptotics and relaxation limit, Meth. Appl. Anal. 19 (2012), 99-118.

[30] A. Schöwe, Global strong solution for large data to the hyperbolic Navier-Stokes equation, arxiv.org/abs/1409.7797 (2014).

[31] J. Serrin, On the uniqueness of compressible fluid motion, Arch. Rational Mech. Anal. 3 (1959), 271-288.

[32] M. Slemrod, Global existence, uniqueness and asymptotic stability of classical smooth solutions in onedimensional non-linear thermoelasticity, Arch. Rational Mech. Anal. 76 (1981), 97-133.

[33] M.A. Tarabek, On the existence of smooth solutions in one-dimensional nonlinear thermoelasticity with second sound, Quart. Appl. Math. 50 (1992), 727-742.

[34] M.E. Taylor, Pseudodifferential operators and nonlinear PDE, Progress Math. 100, Birkhäuser, Boston (1991).

[35] Z.P. Xin, Blowup of smooth solutions to the compressible Navier-Stokes equation with compact density, Comm. Pure. Appl. Math. 51 (1998), 229-240.

[36] W.A. Yong, Entropy and global existence for hyperbolic balance laws, Arch. Rational Mech. Anal. 172 (2004), 247-266.

[37] W.A. Yong, Newtonian limit of Maxwell fluid flows, Arch. Rational Mech. Anal. 214 (2014), 913-922. 